\title{
Water deuterium fractionation in the high-mass hot core G34.26+0.15
}

\author{
F.-C. Liu ${ }^{1 \star \star \star}$, B. Parise ${ }^{1}$, F. Wyrowski ${ }^{1}$, Q. Zhang ${ }^{2}$, and R. Güsten ${ }^{1}$ \\ ${ }^{1}$ Max-Planck-Institut für Radioastronomie, Auf dem Hügel 69, 53121 Bonn, Germany \\ e-mail: fliu@mpifr-bonn.mpg.de \\ 2 Harvard-Smithsonian Center for Astrophysics, 60 Garden Street, Cambridge, MA 02138, USA \\ Received 27 June 2012 / Accepted 9 November 2012
}

\section{ABSTRACT}

\begin{abstract}
Context. Water is an essential molecule in oxygen chemistry and the main constituent of grain icy mantles. The formation of water can be studied through the $\mathrm{HDO} / \mathrm{H}_{2} \mathrm{O}$ ratio. Thanks to the launch of the Herschel satellite and the advance of sensitive submillimeter receivers on ground telescopes, many $\mathrm{H}_{2} \mathrm{O}$ and $\mathrm{HDO}$ transitions can now be observed, enabling more accurate studies of the level of water fractionation.

Aims. Using these new technologies, we aim at revisiting the water fractionation studies toward massive star-forming regions. We present here a detailed study toward G34.26+0.15, a massive star-forming region associated with compact HII regions.

Methods. We present observations of five HDO lines obtained with the APEX telescope. Two of those transitions are ground-state transitions. Two of the three high-excitation lines were additionally observed at higher angular resolution with the SMA. We analyzed these observations using the 1D radiative transfer code RATRAN and adopting different physical profiles from two different models. Results. Although the inner and outer fractional abundances relative to $\mathrm{H}_{2}$ can be best constrained to be $\mathrm{X}_{\text {in }}^{\mathrm{HDO}}(T>100 \mathrm{~K})=$ $(5-7) \times 10^{-8}(3 \sigma)$ and $\mathrm{X}_{\text {out }}^{\mathrm{HDO}}(T \leq 100 \mathrm{~K})=(0.3-2) \times 10^{-11}(3 \sigma)$, the line profile of the $893 \mathrm{GHz}$ ground transition cannot be well reproduced. This line profile is shown to be very sensitive to the velocity field. To better constrain the velocity field, it is necessary to observe the $\mathrm{HDO}$ line at $893 \mathrm{GHz}$ with high angular resolution. The $\mathrm{H}_{2} \mathrm{O}$ abundance is deduced from one high-excitation and one ground transition $\mathrm{H}_{2}^{18} \mathrm{O}$ line. The $\mathrm{D} / \mathrm{H}$ ratios of water are $3.0 \times 10^{-4}$ in the inner region and $(1.9-4.9) \times 10^{-4}$ in the outer region of the core. The HDO fractional abundance in the inner and outer regions are different by more than four orders, which implies that the sublimation is very similar in low- and high-mass protostars. The D/H ratios of water in $\mathrm{G} 34.26+0.15$ are close to the value obtained for the same source in a previous study, and similar to those in other high-mass sources, but lower than those in low-mass protostars, suggesting the possibility that the dense and cold pre-collapse phase is shorter for high-mass star-forming regions.
\end{abstract}

Key words. astrochemistry - stars: massive - stars: protostars - HII regions - ISM: molecules

\section{Introduction}

In the past decades, the abundance of deuterated molecules has been shown to be significantly enhanced in low-temperature environments (e.g. Snell \& Wootten 1977; Wootten et al. 1982; Roueff et al. 2000; Loinard et al. 2001) compared to the D/H elemental ratio in the interstellar medium $\left(\sim 1.5 \times 10^{-5}\right.$, Linsky 2003). The level of deuterium fractionation of molecules is a potentially good tracer of their formation process. This characteristic was demonstrated and used to infer chemical routes of formation for, e.g., methanol (Parise et al. 2004, 2006) and ammonia (Roueff et al. 2005).

Water is an important molecule, because it is the main constituent of grain mantles and an essential molecule in the oxygen chemistry in dense interstellar clouds. It can be formed by three

* Based on observations with the APEX telescope and the SMA. APEX is a collaboration between the Max-Planck-Institut für Radioastronomie, the European Southern Observatory, and the Onsala Space Observatory. The Submillimeter Array (SMA) is a joint project between the Smithsonian Astrophysical Observatory and the Academia Sinica Institute of Astronomy and Astrophysics, and is funded by the Smithsonian Institution and the Academia Sinica.

$\star \star$ Appendix A is available in electronic form at

http: //www . aanda.org

$\star \star \star$ Member of the International Max Planck Research School (IMPRS) for Astronomy and Astrophysics at the Universities of Bonn and Cologne. mechanisms: dissociative recombination in gas phase by ionmolecule chemistry (Bergin et al. 2000), combination of atoms on the surface of cold dust grains at low temperature (Tielens \& Hagen 1982; Cuppen et al. 2010), and gas phase reaction at high temperature (>250 K) (Wagner \& Graff 1987; van der Tak et al. 2006). To constrain the formation process of water, studying the $\mathrm{D} / \mathrm{H}$ ratio of water is a promising method, because the three scenarios would result in different fractionation.

The $\mathrm{D} / \mathrm{H}$ ratio of water has been studied toward several hotcores (Jacq et al. 1990; Pardo et al. 2001; van der Tak et al. 2006) and low-mass protostars (Parise et al. 2005a; Liu et al. 2011). The studies of the high-mass hot cores, Orion IRc 2 and AFGL 2591, and the low-mass protostars, IRAS16293-2422 and NGC1333-IRAS2A, suggest that the HDO emission mainly originates in the envelope and with different $\mathrm{HDO} / \mathrm{H}_{2} \mathrm{O}$ ratios in the two different environments $\left(\sim 10^{-2}\right.$ for low-mass cases and $\sim 10^{-4}$ for high-mass cases). In addition, the $\mathrm{D} / \mathrm{H}$ ratios of water are significantly lower than the formaldehyde and methanol fractionation in the same sources (Parise et al. 2006; Turner 1990), which might be surprising if all species formed simultaneously on dust surfaces. This low deuterium enrichment of water, if confirmed, is a very valuable constraint for astrochemical models that strive to explain the chemical processes involved in the formation of water.

Previous studies have shown that the distribution of the HDO abundance can be constrained in low-mass protostellar 
Table 1. Details of the APEX HDO observations toward G34.26+0.15

\begin{tabular}{lccccccccc}
\hline \hline $\begin{array}{l}\text { Frequency } \\
\mathrm{GHz}\end{array}$ & Transition & $\begin{array}{c}E_{\mathrm{up}} \\
\mathrm{K}\end{array}$ & $\begin{array}{c}n_{\mathrm{cr}}^{\mathrm{a}}\left(\mathrm{ortho}-\mathrm{H}_{2}\right) \\
\mathrm{cm}^{-3}\end{array}$ & $\begin{array}{c}n_{\mathrm{cr}}^{\mathrm{a}}\left(\text { para- } \mathrm{H}_{2}\right) \\
\mathrm{cm}^{-3}\end{array}$ & $\begin{array}{c}\mathrm{HPBW} \\
\prime\end{array}$ & $B_{\text {eff }}$ & $\begin{array}{c}T_{\text {sys }} \\
\mathrm{K}\end{array}$ & $\begin{array}{c}\mathrm{d} v^{b} \\
\mathrm{~km} \mathrm{~s}^{-1}\end{array}$ & Receiver \\
\hline 225.897 & $3_{1,2}-2_{2,1}$ & 167.7 & $1.19 \times 10^{8}$ & $2.65 \times 10^{7}$ & 28 & 0.75 & $196-210$ & 0.16 & APEX-1 \\
241.561 & $2_{1,1}-2_{1,2}$ & 95.3 & $4.00 \times 10^{7}$ & $1.03 \times 10^{7}$ & 26 & 0.75 & $218-235$ & 0.15 & APEX-1 \\
266.161 & $2_{2,0}-3_{1,3}$ & 157.2 & $1.53 \times 10^{9}$ & $3.36 \times 10^{8}$ & 24 & 0.75 & $249-261$ & 0.14 & APEX-1 \\
464.924 & $1_{0,1}-0_{0,0}$ & 22.3 & $1.61 \times 10^{6}$ & $3.74 \times 10^{5}$ & 14 & 0.53 & $498-528$ & 0.08 & PI460 \\
893.639 & $1_{1,1}-0_{0,0}$ & 42.9 & $5.64 \times 10^{7}$ & $1.44 \times 10^{7}$ & 7 & 0.36 & $2656-3685$ & 0.12 & CHAMP $^{+}$ \\
\hline
\end{tabular}

Notes. ${ }^{(a)}$ Critical density at $100 \mathrm{~K}$ calculated with the values of the Einstein coefficient and the collision rates tabulated for HDO (Faure et al. 2012) in the LAMDA database (http: //www. strw. leidenuniv.nl/moldata/). ${ }^{(b)}$ The velocity resolution of the original settings in the observations.

envelopes with the radiative transfer analysis of several HDO lines, which span different energy conditions (Parise et al. 2005b; Liu et al. 2011).

While some detailed studies that also involve the ground transitions of HDO have recently been performed toward lowmass star-forming regions (Parise et al. 2005a; Liu et al. 2011; Coutens et al. 2012), most of the water fractionation studies in high-mass hot cores were performed more than ten years ago, when the submillimeter spectrum was still extremely difficult to observe. Thanks to the advance of sensitive submillimeter receivers, such as those on the APEX telescope, and to the launch of Herschel, it is now possible to carry out much more detailed radiative transfer analyses of $\mathrm{HDO}$ and $\mathrm{H}_{2} \mathrm{O}$. We aim here to carry out such a detailed study on one massive protostellar object, G34.26+0.15, to constrain the formation of water in a highmass hot core.

G34.26+0.15 is a well-studied hot core associated with an ultra-compact $\mathrm{HII}$ region. The $\mathrm{HDO} / \mathrm{H}_{2} \mathrm{O}$ ratio has been derived to be $1.1 \times 10^{-4}$ toward this source from the analysis of one $\mathrm{HDO}$ and one $\mathrm{H}_{2} \mathrm{O}$ transition line (Gensheimer et al. 1996). With the five transition lines including the ground-state lines in the submillimeter-wave range $(241,225,266,464$, and $893 \mathrm{GHz}$ with APEX) and with higher angular resolution observations (241 and $225 \mathrm{GHz}$ with SMA), we can study the HDO abundance and distribution at the core scale. This source was one of the targets of the WISH Key Program (van Dishoeck et al. 2011) with the Herschel telescope, ensuring that information on the abundance of water is available (Wyrowski et al. 2010). This hot core has been the target of molecular line surveys and showed high levels of deuterium fractionation (Hatchell et al. 1998b; Jacq et al. 1990; Gensheimer et al. 1996). The D/H ratios of formaldehyde and HCN toward G34.26 are $\sim 1 \%$ and $\sim 0.1 \%$ (Roberts \& Millar 2007; Hatchell et al. 1998a).

This paper is organized as follows. In Sect. 2 we present the observations. We present the observational results and the radiative transfer modeling in Sects. 3 and 4. The discussion and conclusion are given in Sects. 5 and 6.

\section{Observations}

\subsection{Single-dish observations}

HDO observations were carried out with the APEX telescope. We observed the 225, 241, 266, 464, and $893 \mathrm{GHz}$ lines (Table 1) toward G34.26+0.15 at the hot core position $\alpha_{2000}=$ $18^{\mathrm{h}} 53^{\mathrm{m}} 18^{\mathrm{s}} .57$ and $\delta_{2000}=01^{\mathrm{o}} 14^{\mathrm{m}} 58^{\mathrm{s}} .3$ in September 2010. The focus was checked on Saturn, and the local pointing on G34.26+0.15 itself. We used the wobbler-switching mode with a throw of $240^{\prime \prime}$. Table 1 lists the characteristics of the HDO observations. The temperature scale here was converted from
$T_{\mathrm{A}}^{*}$ to $T_{\mathrm{mb}}$ using the beam efficiencies indicated in Table 1, which we took from the APEX ${ }^{1}$ website. The beam efficiency for $\mathrm{CHAMP}^{+}$was measured in July 2010 on Mars.

\subsection{Interferometer observations}

The observations were carried out on April 26 and May 10, 2011 with the Submillimeter Array (SMA) on Mauna Kea, Hawaii. We used the $230 \mathrm{GHz}$ receivers to observe the 225 and $241 \mathrm{GHz}$ lines as well as the $1.3 \mathrm{~mm}$ continuum simultaneously. The primary-beam size (HPBW) of the 6-m-diameter antennas at $230 \mathrm{GHz}$ was measured to be $\sim 54^{\prime \prime}$. The phase tracking center of this observation is the same as for the APEX observations. The spectral correlator covers $4 \mathrm{GHz}$ bandwidth in each of the two sidebands separated by $10 \mathrm{GHz}$. The frequency coverages are $225.76-229.73 \mathrm{GHz}$ in the lower sideband and 237.73-241.71 GHz in the upper sideband. Each band is divided into 48 chunks. A hybrid resolution mode was set with 512 channels per chunk $(16.2 \mathrm{kHz}$ resolution) for the HDO $J_{\mathrm{K}_{-} \mathrm{K}_{+}}=3_{1,2}-2_{2,1}(\mathrm{~s} 47)$, and 256 channels per chunk $(32.4 \mathrm{kHz})$ for the HDO $J_{\mathrm{K}_{-} \mathrm{K}_{+}}=2_{1,1}-2_{1,2}(\mathrm{~s} 46)$.

The visibility data were calibrated using the MIR software package, which was originally developed for the Owens Valley Radio Observatory. The absolute flux density scale was determined from observations of Neptune for all data. The pair of nearby compact radio sources 1743-038 and 1911-201 was used to calibrate relative amplitude and phase. We used 3C279 to calibrate the bandpass.

The calibrated visibility data were Fourier transformed and CLEANed using the MIRIAD package. The map was made using uniform weighting. The synthesized beam size was 3 . $4 \times 2$ '. 5 with a position angle of $-64^{\circ}$. The continuum map was obtained by averaging all line-free channels in the two bands except chunks s44-s48 because they have different frequency resolution.

\section{First results}

\subsection{Single-dish observations}

The spectra observed toward G34.26+0.15 are presented in Fig. 1. All transitions are detected. They all appear in emission, except for the $893 \mathrm{GHz}$ line, which shows a complex profile, dominated by an absorption component. This is because the $893 \mathrm{GHz}$ line is a ground transition and has a high critical density. The line profiles of the three high-excitation transitions $(225,241$, and $266 \mathrm{GHz})$ are close to Gaussian and their

\footnotetext{
1 http://www . apex-telescope.org/telescope/efficiency/ index.php
} 


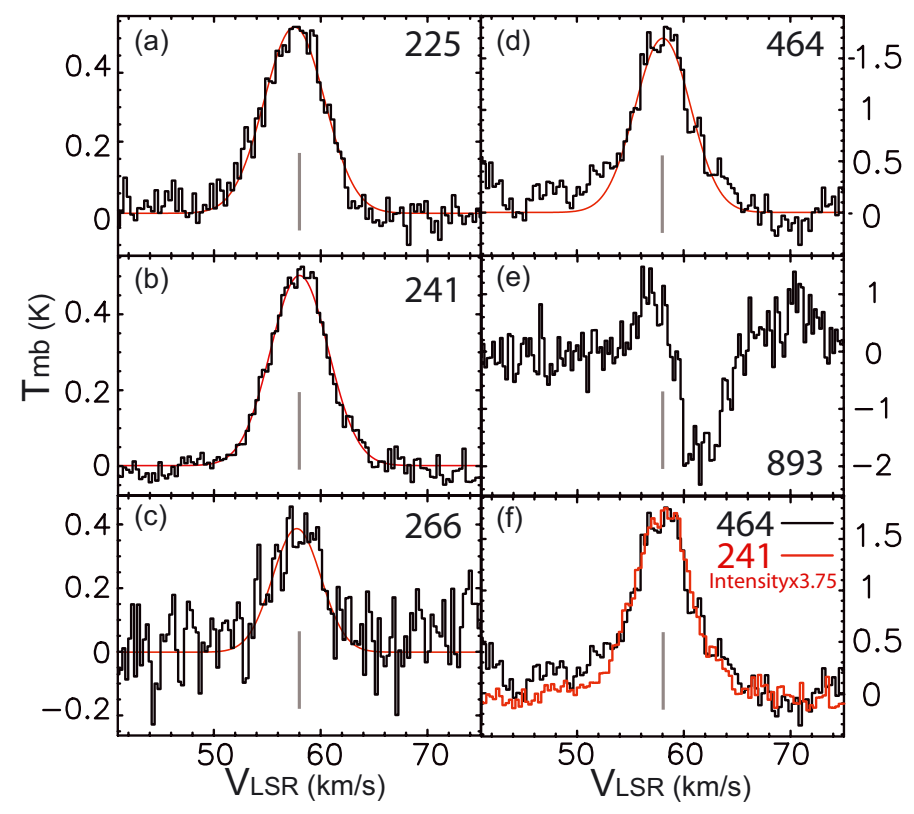

Fig. 1. a)-e) HDO spectra observed with APEX. The red lines indicate the results of the Gaussian fit. f) Comparison of the HDO 464 and $241 \mathrm{GHz}$ spectra. The numbers in the plots indicate the observed frequencies (in GHz). The gray vertical lines are at $V_{\mathrm{LSR}}=58 \mathrm{~km} \mathrm{~s}^{-1}$.

Table 2. Properties of the HDO lines.

\begin{tabular}{ccccccc}
\hline \hline $\begin{array}{c}\text { Freq. } \\
\mathrm{GHz}\end{array}$ & $\begin{array}{c}\mathrm{rms}^{b c e} \\
\mathrm{~K}\end{array}$ & \begin{tabular}{c}
$T_{\text {cont }^{c}} \mathrm{~K}$ \\
\multicolumn{7}{c}{}
\end{tabular} & $\begin{array}{c}T_{\text {peak }}^{c e} \\
\mathrm{~K}\end{array}$ & $\begin{array}{c}V_{\mathrm{LSR}^{e}} \\
\mathrm{~km} \mathrm{~s}^{-1}\end{array}$ & $\begin{array}{c}\Delta v^{d e} \\
\mathrm{~km} \mathrm{~s}^{-1}\end{array}$ & $\begin{array}{c}\int T_{\mathrm{mb}} \mathrm{d} v^{e f} \\
\mathrm{~K} \mathrm{~km} \mathrm{~s}^{-1}\end{array}$ \\
\hline 225 & 0.06 & 0.85 & 0.53 & $57.5 \pm 0.1$ & $6.7 \pm 0.2$ & $3.8 \pm 0.1$ \\
241 & 0.04 & 0.88 & 0.52 & $58.0 \pm 0.2$ & $6.3 \pm 0.5$ & $3.5 \pm 0.2$ \\
266 & 0.12 & 0.9 & 0.37 & $57.9 \pm 0.2$ & $6.6 \pm 0.6$ & $2.6 \pm 0.2$ \\
464 & 0.24 & 4.0 & 1.66 & $57.9 \pm 0.2$ & $7.2 \pm 0.4$ & $12.6 \pm 0.6$ \\
893 & 0.53 & 13.0 & $-1.89^{a}$ & $61.9 \pm 0.1^{a}$ & $3.7 \pm 0.1^{a}$ & $-8.4 \pm 0.6^{a}$ \\
\hline \multicolumn{7}{c}{ SMA observations } \\
\hline 225 & 4.2 & $\ldots$ & 0.32 & $57.9 \pm 0.1$ & $7.0 \pm 0.2$ & $2.4 \pm 0.1$ \\
241 & 17.0 & $\cdots$ & 0.33 & $57.9 \pm 0.1$ & $6.3 \pm 0.2$ & $2.2 \pm 0.1$ \\
\hline \multicolumn{7}{c}{}
\end{tabular}

Notes. (a) Only the absorption part, between velocity ( $\left.V_{\mathrm{LSR}}\right)$ $57.6-66.2 \mathrm{~km} \mathrm{~s}^{-1}$. ${ }^{(b)}$ The rms values are measured with the resolutions of the original observation settings (Table 1). ${ }^{(c)} T_{\mathrm{mb}}$ scale. ${ }^{(d)}$ FWHM. (e) Value is derived from a Gaussian fit using the software CLASS. (f) The uncertainties only include the statistical noise and not the calibration uncertainties.

linewidths are $\sim 6.3-6.7 \mathrm{~km} \mathrm{~s}^{-1}$, obtained from a Gaussian fit. Not only are the profiles of 225 and $241 \mathrm{GHz}$ HDO lines very similar, but their intensities are very close to each other. The linewidth of the $464 \mathrm{GHz}$ HDO line is slightly wider than the high-excitation lines $\left(\sim 7.2 \mathrm{~km} \mathrm{~s}^{-1}\right)$, although this does not appear to be significant, owing to the large error bars, and as shown in Fig. 1f, which displays the superposition of the 464 and $241 \mathrm{GHz}$ line profiles. It is found that the difference between these two spectra is still within the $3 \sigma$ noise level, and that there is no obvious evidence for the existence of a broad component in the $464 \mathrm{GHz}$ line, as was found in some ground transition $\mathrm{H}_{2} \mathrm{O}$ lines (Kristensen et al. 2010b; Chavarría et al. 2010).

Table 2 lists the properties of the observed HDO lines. Using the integrated line intensities, we performed a rotation diagram analysis assuming that the lines are optically thin and in local

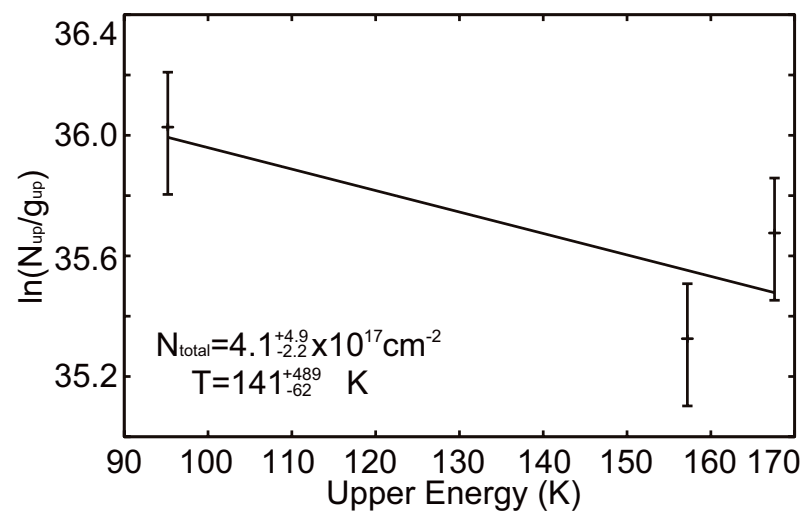

Fig. 2. HDO rotation diagram. The detected lines are shown as crosses with error bars. The size of the error bars corresponds to $20 \%$ of the intensity due to the calibration uncertainty.

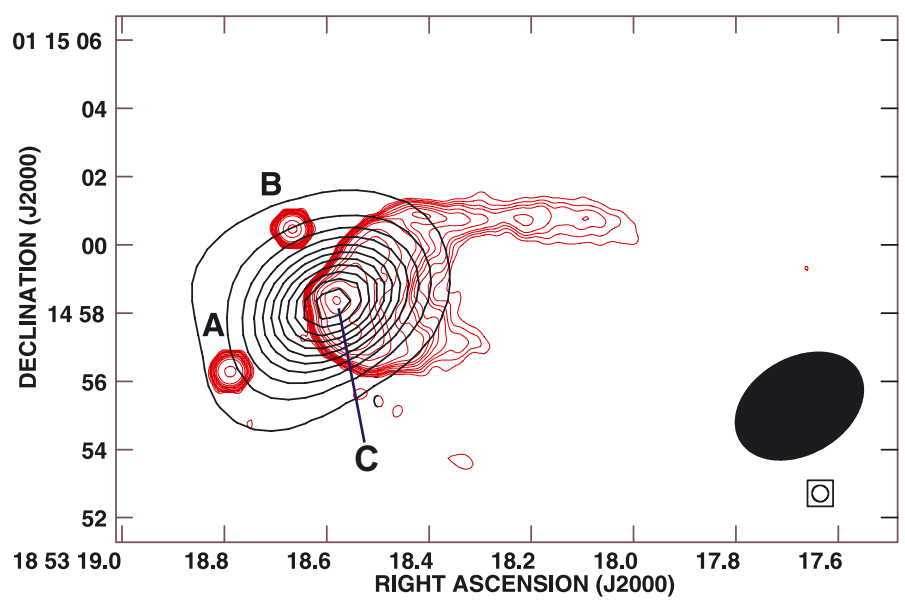

Fig. 3. Overlay of the $1.3 \mathrm{~mm}$ continuum emission observed with the SMA (black contours) with the red contours of the $43.3 \mathrm{GHz}$ free-free continuum emission observed by Avalos et al. (2006). Contour levels are from $3 \sigma$ by steps of $6 \sigma$, with $1 \sigma=0.1 \mathrm{Jy} \mathrm{beam}^{-1}$ for the SMA images and at $-4,-3,3,4,5,6,8,10,12,15,20,40,60,100,200,400$, and 800 times the noise, with $1 \sigma=1.04$ mJy beam $^{-1}$ for the $43.3 \mathrm{GHz}$ images. The synthesized beams of the SMA (black) and free-free emission (red) images are shown in the lower right of the plot. The synthesized beam of the $2-\mathrm{cm}$ image is $1^{\prime \prime} .00 \times 00^{\prime} .85$ with $\mathrm{PA}=35^{\circ}$.

thermodynamic equilibrium (LTE) (Goldsmith \& Langer 1999). We considered only the three high-excitation lines, because the two ground-state lines are suggested to be optically thick (see Sect. 4.3). We assumed a $1^{\prime \prime}$ source size, as suggested from the interferometric observations (Table 3). Because this size is smaller than the three observational beams, the derived column density and source size are degenerate, and the temperature does not depend on the size assumption. The resulting rotation diagram is displayed in Fig. 2. The rotation temperature obtained is $\sim 141_{-62}^{+489} \mathrm{~K}$, which is consistent with the estimates of the kinetic temperature $(160 \pm 30 \mathrm{~K})$ from the analysis of $\mathrm{OCS}, \mathrm{CH}_{3} \mathrm{OH}$, $\mathrm{SO}$, and $\mathrm{HC}_{3} \mathrm{~N}$ (Mookerjea et al. 2007). The derived total molecular column density of the hot HDO is $4.1 \times 10^{17} \mathrm{~cm}^{-2}$ averaged on the assumed $1^{\prime \prime}$ extended source.

\subsection{Interferometer observations}

\subsubsection{Continuum at $\lambda=1.3 \mathrm{~mm}$}

The total continuum flux retrieved within the SMA primary beam is $8.0 \mathrm{Jy}$. The continuum emission is found to be slightly 
Table 3. Results of the SMA observations

\begin{tabular}{|c|c|c|c|c|c|c|c|c|c|}
\hline & \multirow{2}{*}{$\underset{\prime \prime}{\text { Beam }}$} & \multirow{2}{*}{$\begin{array}{l}\text { Freq. } \\
\text { GHz }\end{array}$} & \multirow{2}{*}{$\begin{array}{c}\mathrm{rms} \\
\text { Jy/Beam }\end{array}$} & \multirow[t]{2}{*}{ Integrated flux $^{b}$} & \multicolumn{2}{|c|}{ Peak position $^{b}$} & \multirow{2}{*}{\multicolumn{2}{|c|}{$\underset{\prime \prime}{\text { Positional error }}{ }^{c}$}} & \multirow{2}{*}{$\underset{\prime \prime}{\text { Deconvolved size }}$} \\
\hline & & & & & $\mathrm{RA}(\mathrm{J} 2000)$ & $\operatorname{Dec}(J 2000)$ & & & \\
\hline HDO 225 & 3." $70 \times 2$ 2. 54 & 225.9 & 0.06 & $71.3 \mathrm{Jy} \mathrm{km} \mathrm{s}^{-1}$ & $18^{\mathrm{h}} 53^{\mathrm{m}} 18^{\mathrm{s}} .61$ & $01^{\circ} 14^{\mathrm{m}} 58^{\mathrm{s}} .38$ & 0.04 & 0.02 & $\ldots{ }^{a b}$ \\
\hline HDO 241 & 3 3. $^{\prime} 57 \times 2$ '. 44 & 241.6 & 0.09 & $72.8 \mathrm{Jy} \mathrm{km} \mathrm{s}^{-1}$ & $18^{\mathrm{h}} 53^{\mathrm{m}} 18^{\mathrm{s}} .61$ & $01^{\circ} 14^{\mathrm{m}} 58^{\mathrm{s}} .46$ & 0.04 & 0.02 & $1^{\prime \prime} 12 \times 00^{\prime \prime} 67$ \\
\hline $1.3 \mathrm{~mm}$ & 3." $44 \times 2$ '. 35 & 233.6 & 0.05 & $8.0 \mathrm{Jy}$ & $18^{\mathrm{h}} 53^{\mathrm{m}} 18^{\mathrm{s}} .59$ & $01^{\circ} 14^{\mathrm{m}} 58^{\mathrm{s}} .22$ & 0.02 & 0.02 & $1^{\prime \prime} 51 \times 11^{\prime \prime} 03$ \\
\hline
\end{tabular}

Notes. ${ }^{(a)}$ Deconvolution appears to produce a point source. ${ }^{(b)}$ Values are obtained from a Gaussian fit using the task IMFIT of the software MIRIAD. ${ }^{(c)}$ Values are derived from the theoretical formula $\sigma_{\text {position }}=F W H M /[\sqrt{8 \ln 2}(S / N)]$ (Condon 1997).

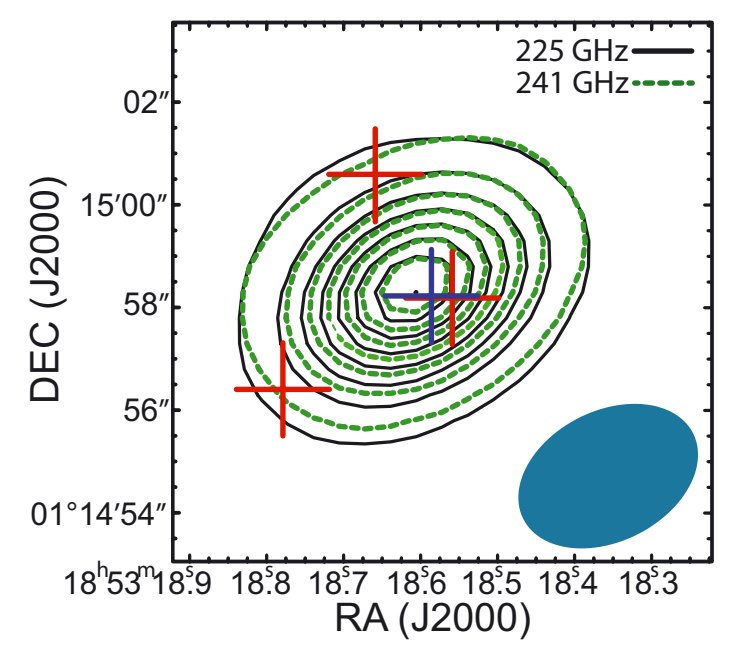

Fig. 4. HDO $225 \mathrm{GHz}$ (black contours) and HDO $241 \mathrm{GHz}$ (greendashed contours) integrated map observed with the SMA. The contour levels are from $3 \sigma$ by steps of $6 \sigma$, with $1 \sigma=1.5 \mathrm{Jy} \mathrm{beam}^{-1}$. The red crosses indicate the peak of the $24 \mathrm{GHz}$ free-free continuum emission (A, B, and C positions from Heaton et al. 1989) and the peak (maximum pixel) of the $1.3 \mathrm{~mm}$ continuum observed with the SMA is shown with the dark blue cross. The blue ellipse indicates the beam size.

resolved (Table 3). Figure 3 shows an overlay of the $1.3 \mathrm{~mm}$ continuum image with the free-free continuum emission observed by Avalos et al. (2006). The continuum peak detected at $1.3 \mathrm{~mm}$ does not coincide with HII regions $\mathrm{A}$ and $\mathrm{B}$, but is roughly consistent with $\mathrm{HII}$ region $\mathrm{C}$ within the beam size (detail see Fig. 4). At $107 \mathrm{GHz}$, the integrated continuum intensity of $6.7 \mathrm{Jy}$ is suggested to be dominated by free-free emission and only $\sim 10 \%$ can be contributed from dust (Mookerjea et al. 2007). To date, neither the turnover point of free-free emission nor the spectral index are clear. Thus, if the free-free emission is already optically thin at a wavelength longer than $3 \mathrm{~mm}$, the freefree emission will contribute $\sim 6 \mathrm{Jy}$ to the emission at $1.3 \mathrm{~mm}$. In other words, the detected continuum flux with the SMA is the combination of free-free and dust emission. Figure 4 also supports this argument, because the peak of the $1.3 \mathrm{~mm}$ continuum is located between the peak of the free-free emission (from which it is offset by $\sim 0.3^{\prime \prime}$ ) and the HDO peak. This point will be important when modeling the emission with RATRAN (which models only the dust continuum).

\subsubsection{Spectral lines}

The observational results for the two HDO lines are listed in Table 3. The integrated intensity maps of the two HDO lines (Fig. 4) show that their emission distributions are similar, suggesting that the emission comes from the same gas. In addition, the emission is not coincident with the A and B compact
HII regions. The peak of the HDO emission is offset from the $1.3 \mathrm{~mm}$ dust continuum peak by approximately $0.3-0.4^{\prime \prime}$ (Table 3), under the theoretically positional error of approximately $0.04^{\prime \prime}$ with a signal-to-noise ratio $(\mathrm{S} / \mathrm{N})$ of 43 . We believe that this offset is significant, because the continuum and the lines were observed simultaneously. This offset seems to be much smaller than the offset $\left(2^{\prime \prime}\right)$ observed for $\mathrm{CH}_{3} \mathrm{CN}$ by Watt \& Mundy (1999), and on the lower end of the different offsets observed for different molecules $\left(\mathrm{CH}_{3} \mathrm{OH}, \mathrm{SO}\right.$, and OCS) by Mookerjea et al. (2007). Those molecules have lower critical densities $^{2}$ compared to the two HDO lines, so excitation effects might partly explain the discrepancy between the distribution of the molecules.

Comparing the results observed with APEX, we find that the missing flux of the HDO $225 \mathrm{GHz}$ and $241 \mathrm{GHz}$ line emission are $\sim 37.1 \%$ and $\sim 36.4 \%$, suggesting that most HDO emission is coming from the center of the molecular cloud core, which is consistent with the fact that the line shapes are very similar. Here, the SMA map was convolved with the beam size of APEX. The calibration uncertainties of APEX and SMA are usually considered to be $\sim 20 \%$ and $5-15 \%$. It is difficult to believe that the apparent missing flux is real, because that would require that these high-excitation HDO lines show some extended emission, which is very unlikely in view of their critical density and energy. Indeed, the differences in fluxes between the SMA and APEX spectra can be also caused by the side lobes in the SMA images. Because the declination $\left(\delta_{2000}\right)$ of our source is around 1 degree, the side lobes are quite strong and cannot be easily removed thoroughly. The negative side lobes will lower the obtained integrated flux (SMA) in this case. We therefore assume that the flux discrepancy is only originating in the uncertainty of the calibration and imaging of the data.

We resolved the CN 2-1 hyperfine structure of $J=3 / 2-1 / 2$ and $J=5 / 2-3 / 2$. All $\mathrm{CN}$ absorption lines show redshifted absorption; we discuss in more detail in Sect. 5.2.2.

\section{Modeling}

\subsection{Physical profiles of the source}

To analyze the HDO data, we used the 1 D Monte Carlo code RATRAN, developed by Hogerheijde \& van der Tak (2000). The radiative transfer model takes as input the physical $(n, T)$ profiles of the source. The heating source of the hot core and its density structure are not clearly understood toward G34.26+0.15, because there is no high angular resolution study $\left(\leq 1^{\prime \prime}\right)$ in the submillimeter band. It has been argued that the hot core could be

2 At $100 \mathrm{~K}$, their critical densities are $1.1 \times 10^{5}, 3.8 \times 10^{6}, 6.6 \times$ $10^{4}$, and $5.3 \times 10^{3} \mathrm{~cm}^{-3}$, calculated using the values of the Einstein coefficient and the collision rates tabulated (Green 1986; Pottage et al. 2004; Kristensen et al. 2010a; Green 1994; Green \& Chapman 1978; Schöier et al. 2005) in the LAMDA database. 


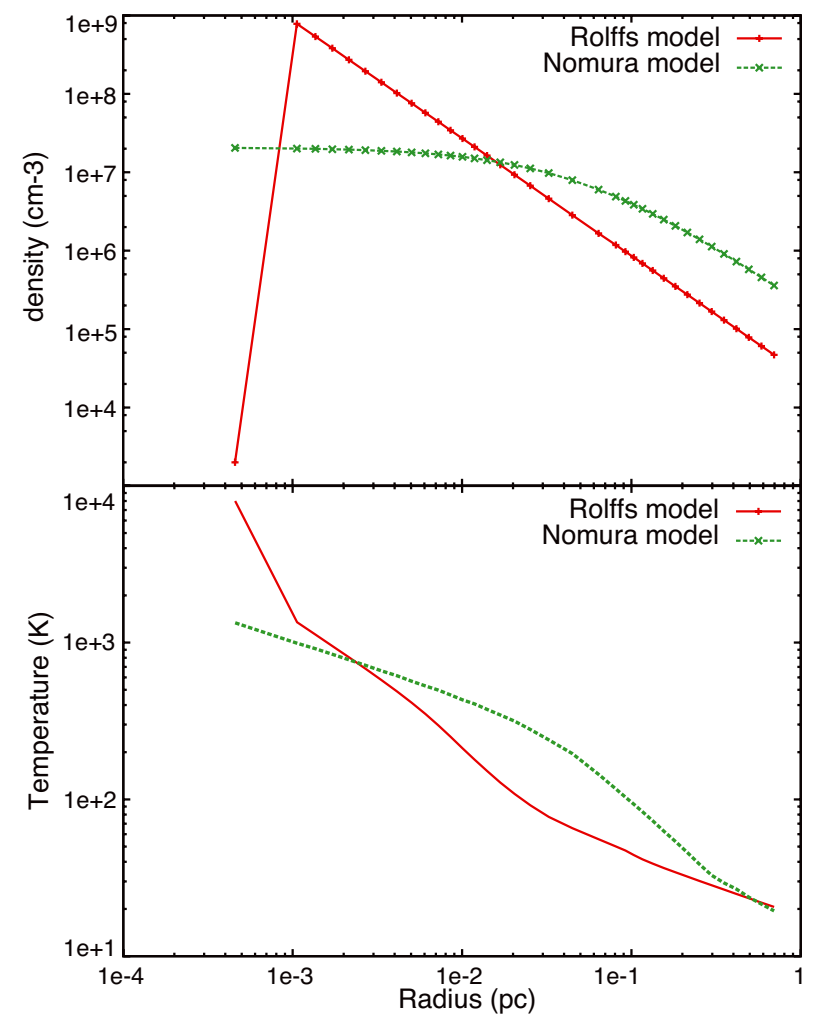

Fig. 5. Comparison of the physical profiles of the two different models toward G34.26+0.15 (Nomura \& Millar 2004; Rolffs et al. 2011).

externally heated by this HII region (Mookerjea et al. 2007), because the hot core lies off the cometary-shaped HII region (Watt \& Mundy 1999). This cometary-shape may be caused by the bow shock produced by the wind from a young star that moves at supersonic speed through an ambient molecular cloud (Wood \& Churchwell 1989; van Buren et al. 1990; Watt \& Mundy 1999) or by the champagne outflow (Andersson \& Garay 1986; Fey et al. 1992; Carral \& Welch 1992; Gaume et al. 1994). The separations between the HII region and the emission peaks of most $\mathrm{N}$ - and O-bearing molecules are $\leq 1^{\prime \prime}$ (Mookerjea et al. 2007, see Fig. 3). However, recent studies with $\mathrm{HCN}, \mathrm{HCO}^{+}, \mathrm{CO}$, and their isotopes observed with APEX show no evidence for this scenario and, moreover, the results show self-absorption and strong red asymmetries, which suggest an in-falling source with internal heating (Rolffs et al. 2011). In addition, the studies of the $\mathrm{NH}_{3}$ $\left(3_{2}-2_{2}\right)$ line observed with SOFIA also present a clear in-fall absorption in the spectra which are well reproduced also with Rolffs' model (Wyrowski et al. 2012). This may be because they probe larger scales and the structures of the heating area are too small to be resolved in their observations (Rolffs et al. 2011). Therefore, the external heating structures could be internal in the model. Neither our APEX nor our SMA data have a sufficiently high resolution to probe the scale of the heating region; therefore, the two models we adopted here have the temperature structures derived by the inner heating source.

We use in the following the physical profiles of two different models (Nomura \& Millar 2004; Rolffs et al. 2011, Fig. 5). Nomura's model is constrained by the observed spectral energy distribution (SED) data at wavelengths from far-infrared to millimeter. Most data are from Chini et al. (1987) and were observed with the ESO $1 \mathrm{~m}$ telescope, except for an additional point at around $3 \mathrm{~mm}$ (Nomura \& Millar 2004, see Fig. 1c). The 3-mm data point is taken from Watt \& Mundy (1999) and were observed with BIMA (Nomura, private communication). As in Nomura's study, the model with the column density of $N=10^{25} \mathrm{~cm}^{-2}$ is used here. The density and temperature profiles of the Rolffs model aim to reproduce the radial intensity profile from LABOCA data (345 GHz continuum). We adopted the opacity and velocity field setting of the Rolffs model for both models (opacity: ice mantle coagulation at a density of $10^{5} \mathrm{~cm}^{-3}$, Ossenkopf \& Henning 1994; velocity field: $V=$ $-0.2\left(\frac{2 G M_{\text {in }}}{r}\right)^{1 / 2}$, Rolffs et al. 2011). The turbulent linewidth, $\mathrm{db}$, is set to be $3.0 \mathrm{~km} \mathrm{~s}^{-1}$. The adopted distance is $3.7 \mathrm{kpc}$ (Kuchar $\&$ Bania 1994). The HDO collisional rates used in this study are computed by Faure et al. (2012) for para- $\mathrm{H}_{2}$ and ortho- $\mathrm{H}_{2}$ separately. In the modeling, we assumed that the ortho-to-para ratio of $\mathrm{H}_{2}$ is in LTE in each cell of the core.

\subsection{Modeling procedure}

The HDO emission was modeled with a jump model, where the fractional abundances of deuterated water, relative to $\mathrm{H}_{2}$, in the inner part of the source ( $T>100 \mathrm{~K}$, assumed evaporation temperature) and in the outer part $(T \leq 100 \mathrm{~K})$ are two free parameters, $\mathrm{X}_{\text {in }}^{\mathrm{HDO}}$ and $\mathrm{X}_{\mathrm{out}}^{\mathrm{HDO}}$. We made two sets of comparisons, one with the APEX data and the other with the SMA data. For comparison with the APEX data, the modeled maps were convolved with the APEX beam. For comparison with the SMA data, we used MIRIAD to create mock observations based on the modeled data. We first inserted the values of the observed parameters (epoch, rest frequency, and the coordinate of the observed reference position) into the blank header of the modeled data. Then, we multiplied the modeled image by the SMA primary beam. Afterward, we applied the $(u, v)$ sampling retrieved from the SMA data to the modeled image and inverted the resulting $(u, v)$ data to obtained the simulated maps/spectra. We then performed $\chi^{2}$ analyses for $\mathrm{X}_{\mathrm{in}}^{\mathrm{HDO}}$ ranging from $1 \times 10^{-9}$ to $4 \times 10^{-7}$ and for $\mathrm{X}_{\text {out }}^{\mathrm{HDO}}$ ranging from $1 \times 10^{-15}$ to $2 \times 10^{-11}$ for the Rolffs model and for $\mathrm{X}_{\mathrm{in}}^{\mathrm{HDO}}$ ranging from $1 \times 10^{-12}$ to $5 \times 10^{-9}$ and for $\mathrm{X}_{\mathrm{out}}^{\mathrm{HDO}}$ ranging from $1 \times 10^{-17}$ to $5 \times 10^{-10}$ for the Nomura model. We intend to also model the line velocity profiles and all spectra, including the spectra from the SMA data. Here the definition of $\chi^{2}$ is $\Sigma \frac{\left(T_{\mathrm{mb}, \mathrm{ob}}-T_{\mathrm{mb}, \mathrm{mod}}\right)^{2}}{\sigma^{2}}$, where the sum is over each channel in each spectrum. The $\sigma$ within this $\chi^{2}$ analysis includes the statistical errors and uncertainties in flux calibration $(\sim 10 \%$ for 225 and $241 \mathrm{GHz}$ observed with SMA, $30 \%$ for $893 \mathrm{GHz}$ and $20 \%$ for other lines observed with APEX), but does not include any uncertainty in the adopted collisional rate coefficients used in the excitation calculation (Faure et al. 2012).

\subsection{Results}

The black solid lines in Fig. 6 present the contours delimitating the $1 \sigma(68.3 \%), 2 \sigma(95.4 \%)$ and $3 \sigma(99.7 \%)$ confidence intervals. These contours are derived with the method described in Lampton et al. (1976), who showed that the $\Delta S=\chi^{2}-\chi_{\text {min }}^{2}$ random variable follows a $\chi^{2}$ distribution with $p$ variables, $p$ being the number of parameters. Here $p=2\left(\mathrm{X}_{\text {in }}\right.$ and $\left.\mathrm{X}_{\text {out }}\right)$, so that these contours correspond to $\chi^{2}=\chi_{\min }^{2}+2.3, \chi_{\min }^{2}+6.17$ and $\chi_{\min }^{2}+11.8$. Here we used $1 \sigma, 2 \sigma$, and $3 \sigma$ in analogy with Gaussian-distributed random variables.

The fitting parameters and obtained results are listed in Table 4. The observed spectra are overlaid with the modeling results of the best fit in Fig. 7. Here the system velocity of $58 \mathrm{~km} \mathrm{~s}^{-1}$ is assumed $\left(V_{\mathrm{LSR}}\right)$. The minimum $\chi_{\text {red }}^{2}$ are not close 
Table 4. Fitting parameters and their results.

\begin{tabular}{lcclllll}
\hline \hline Physical profiles & Turbulent linewidth $^{a}$ & Radius at 100 K & Best fit HDO abundances & $\chi_{\text {red }}^{2}$ & HDO abundances confidence interval $(3 \sigma)$ \\
\hline & $\mathrm{km} \mathrm{s}^{-1}$ & $\mathrm{pc}$ & inner region & outer region & inner region & outer region \\
\hline Rolffs model $^{b}$ & 3.0 & 0.02 & $6.0 \times 10^{-08}$ & $5.0 \times 10^{-12}$ & 6.0 & $(5.0-7.0) \times 10^{-08}$ & $(0.3-2.0) \times 10^{-11}$ \\
Nomura model $^{c}$ & 3.0 & 0.09 & $4.0 \times 10^{-10}$ & $1.0 \times 10^{-14}$ & 19.6 & $(2.0-5.0) \times 10^{-10}$ & $\leq 1 \times 10^{-13}$ \\
\hline
\end{tabular}

Notes. ${ }^{(a)}$ Fixed parameter. ${ }^{(b)}$ Rolffs11. ${ }^{(c)}$ Nomura \& Millar (2004).
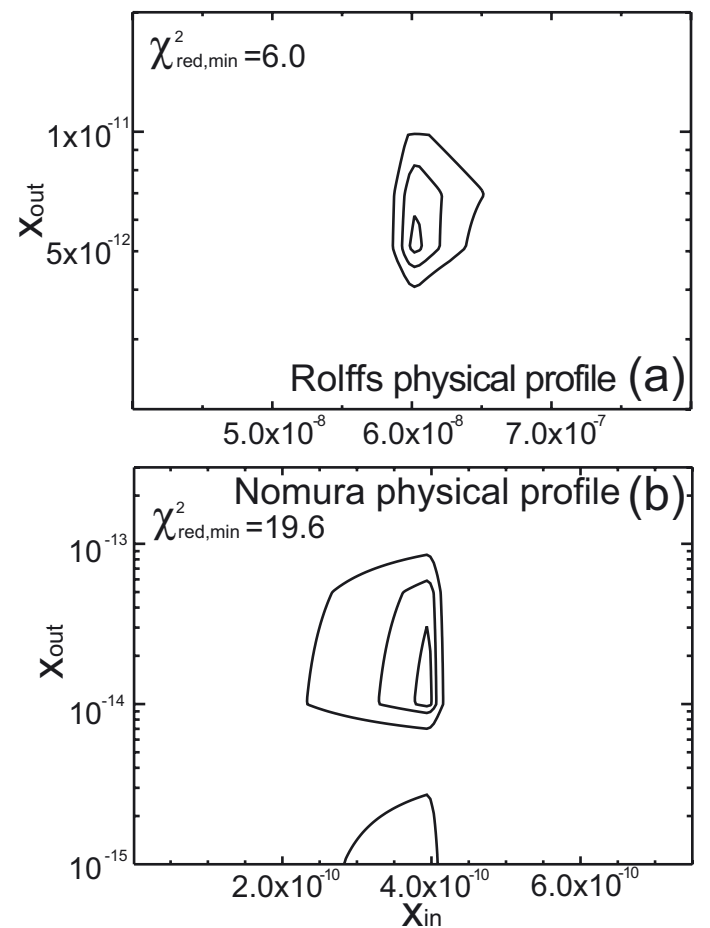

Fig. 6. $X_{\text {in }}^{\mathrm{HDO}}$ and $\mathrm{X}_{\text {out }}^{\mathrm{HDO}}$ contours $(1,2$ and $3 \sigma)$ for the $\chi_{\text {red }}^{2}$ for the different models. a) and b) show the $\chi^{2}$ contours calculated over all spectra with the Rolffs and Nomura physical profiles.

to 1 , which indicates that the spectra are not well reproduced by the two different physical models. This is also obvious in Fig. 7. When the Rolffs model is adopted (red lines in Fig. 7), the emission at 225, 241, and $266 \mathrm{GHz}$ is not high enough at the large scale to fit the observed data (APEX data), but the most compact emission in the 225 and $241 \mathrm{GHz}$ lines is well reproduced (SMA data). At the same time, the simulated spectra at 464 and $893 \mathrm{GHz}$ are optically thick $(\tau=2.1$ and $\tau=24.1$ at lines and source center) and the line profile in $893 \mathrm{GHz}$ spectrum does not fit the observed spectrum either. In Fig. 7, the blue and green lines indicate the results of the best-fit model using the Nomura physical profile, the green line was obtained excluding the spectrum at $893 \mathrm{GHz}$ from the $\chi^{2}$ analysis. In this case, the fractional abundances are $2 \times 10^{-9}$ and $5 \times 10^{-12}$ for the inner and outer region. This model fails to reproduce the central emission observed with the SMA.

Comparing the results reproduced by the two models (red and blue), we find that the best fit based on the Rolffs model is closer to the observed data $\left(\chi_{\text {red }}^{2}=6.0\right)$. The fitting problems in the ground transitions (464 and $893 \mathrm{GHz}$ ) are slightly less severe than for the Nomura model (see Fig.7). Moreover, from the comparison of the two fitting results of the Nomura model (blue and green), we find that the absorption in the $893 \mathrm{GHz}$
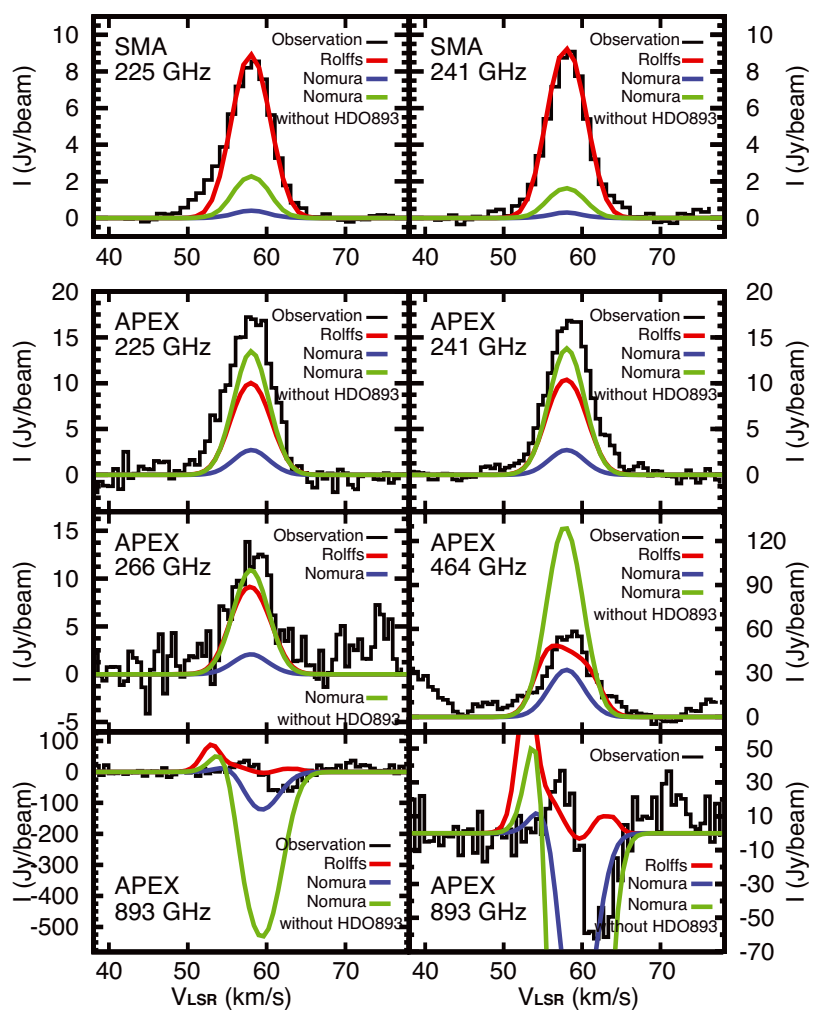

Fig. 7. Comparison of the observed spectra with the results of our bestfit models for the two different physical profiles. The observed line emission is shown as black histograms in each spectrum. The red, green, and blue lines show the best-fit results of the Rolffs model, the Nomura model with all spectra, and the Nomura model excluding the HDO spectrum at $893 \mathrm{GHz}\left(\mathrm{X}_{\mathrm{in}}^{\mathrm{HDO}}=2 \times 10^{-9} ; \mathrm{X}_{\mathrm{out}}^{\mathrm{HDO}}=5 \times 10^{-12}\right)$.

spectra and emission in $464 \mathrm{GHz}$ are too strong if we try to increase the emission in the high-excitation lines to fit the spectra $(225,241,266 \mathrm{GHz})$. Indeed, the continuum flux reproduced with the Nomura model $(\sim 49 \mathrm{~K})$ is much higher than the real flux $(\sim 13.0 \mathrm{~K})$. This is also found in Appendices A.2. and A.3. Figure 8 shows the angular distribution of the $225 \mathrm{GHz}$ line from the SMA observation and the two models. Here again the model based on the Rolffs profiles fits the data much better. This is similar for the $241 \mathrm{GHz}$ line and is therefore not shown.

The model based on the Rolffs centrally heated core reproduces the SMA observed line distribution well. This does not exclude the possibility that the heating of the very central dust core is external, but it shows that even at the resolution of our SMA data, we do not see clear evidence of a non-spherically symmetric heating of the envelope, and that the large-scale properties of the envelope can therefore be derived using such a model. Distinguishing between internal and external heating at small scales would require observations with much higher angular resolution. 
F.-C. Liu et al.: water deuterium fractionation in G34.26+0.15

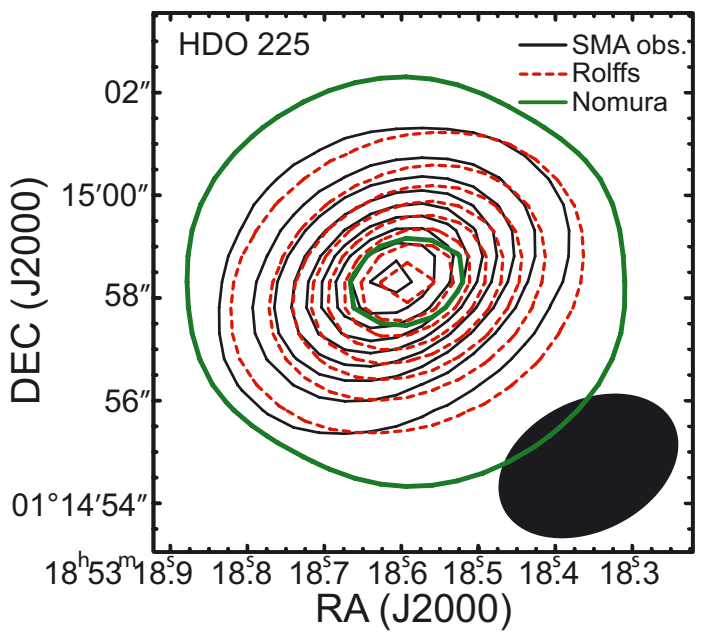

Fig. 8. Overlay of the HDO $225 \mathrm{GHz}$ integrated map observed with the SMA (black contours) with the simulated ones of the best-fit results of the Rolffs model and of the Nomura model excluding the HDO spectrum at $893 \mathrm{GHz}$. The red and green contours show the integrated HDO $225 \mathrm{GHz}$ map of the best-fit results of the Rolffs model and of the Nomura model excluding the HDO spectrum at $893 \mathrm{GHz}$. These two integrated maps are simulated to be observed with the uv-coverage of the SMA observations. All contour levels are from $3 \sigma$ by steps of $5 \sigma$, with $1 \sigma=1.5 \mathrm{Jy} \mathrm{beam}^{-1}$. The black ellipse indicates the beam size.

Based on the superior results obtained with the Rolffs profiles, we focus on the Rolffs model below and try to reproduce the lines with a two-jump model of the HDO abundance distribution. Finally, we modify Rolffs physical structure to try to fit the observed data. The results of the two-jump model and modified models are discussed in Sects. 5.1 and 5.2 and in Appendices A.2. and A.3.

\section{Discussion}

\subsection{Two-jump model}

To reproduce more emission in the high-excitation lines and decrease the emission in the $893 \mathrm{GHz}$ line, we modeled the spectra with a two-jump model instead of a one-jump model. The additional jump was arbitrarily set to be located at $T=200 \mathrm{~K}$. Figure 9 show the results of four testing models. The adopted HDO fractional abundances of the models are listed in Table 5. The red lines indicate the results of the original best-fit one-jump model, which underestimates the high-excitation lines. The twojump models (2J-1 and 2J-3) are found to be somewhat better for these transitions (Fig. 9). This suggests that the two-jump model can produce higher emission in the high-excitation lines. This property has already been found for Sgr B2 (M) (Comito et al. 2010) and this is due to the very high critical density of these lines (see Table 1). Moreover, although we decreased the HDO fractional abundance in the second inner region of the core in model $2 \mathrm{~J}-2$ and $2 \mathrm{~J}-4$ by a factor of 2 , the emission in the $893 \mathrm{GHz}$ line is little affected $(<1 \sigma)$. In addition, the line shapes of different lines do not change from model to model. Therefore, the results suggest that adopting a two-jump model can help in increasing the emission in the high-excitation lines, but cannot improve the 893 and $464 \mathrm{GHz}$ line shapes. We note, however, that because the inner part is very compact, the enhancement of the high-excitation lines in the APEX beam when increasing the HDO abundance in this inner part is linked to an even faster relative enhancement of the flux in the SMA beam.
Table 5. Parameters of the models.

\begin{tabular}{lccc}
\hline \hline Model & $\mathrm{X}_{\mathrm{in1}}^{\mathrm{HDO}}$ & $\mathrm{X}_{\mathrm{in2}}^{\mathrm{HDO}}$ & $\mathrm{X}_{\text {out }}^{\mathrm{HDO}}$ \\
\hline One jump $^{a}$ & $6 \times 10^{-8}$ & $6 \times 10^{-8}$ & $5 \times 10^{-12}$ \\
\hline Two jump 1 (2J1) & $3 \times 10^{-7}$ & $6 \times 10^{-8}$ & $5 \times 10^{-12}$ \\
Two jump 2 (2J2) & $3 \times 10^{-7}$ & $3 \times 10^{-8}$ & $5 \times 10^{-12}$ \\
Two jump 3 (2J3) & $2 \times 10^{-7}$ & $6 \times 10^{-8}$ & $5 \times 10^{-12}$ \\
Two jump 4 (2J4) & $2 \times 10^{-7}$ & $3 \times 10^{-8}$ & $5 \times 10^{-12}$ \\
Two jump 5 (2J5) & $2 \times 10^{-7}$ & $3 \times 10^{-8}$ & $1 \times 10^{-14}$ \\
\hline
\end{tabular}

Notes. ${ }^{(a)}$ The results of the one-jump best-fit model.
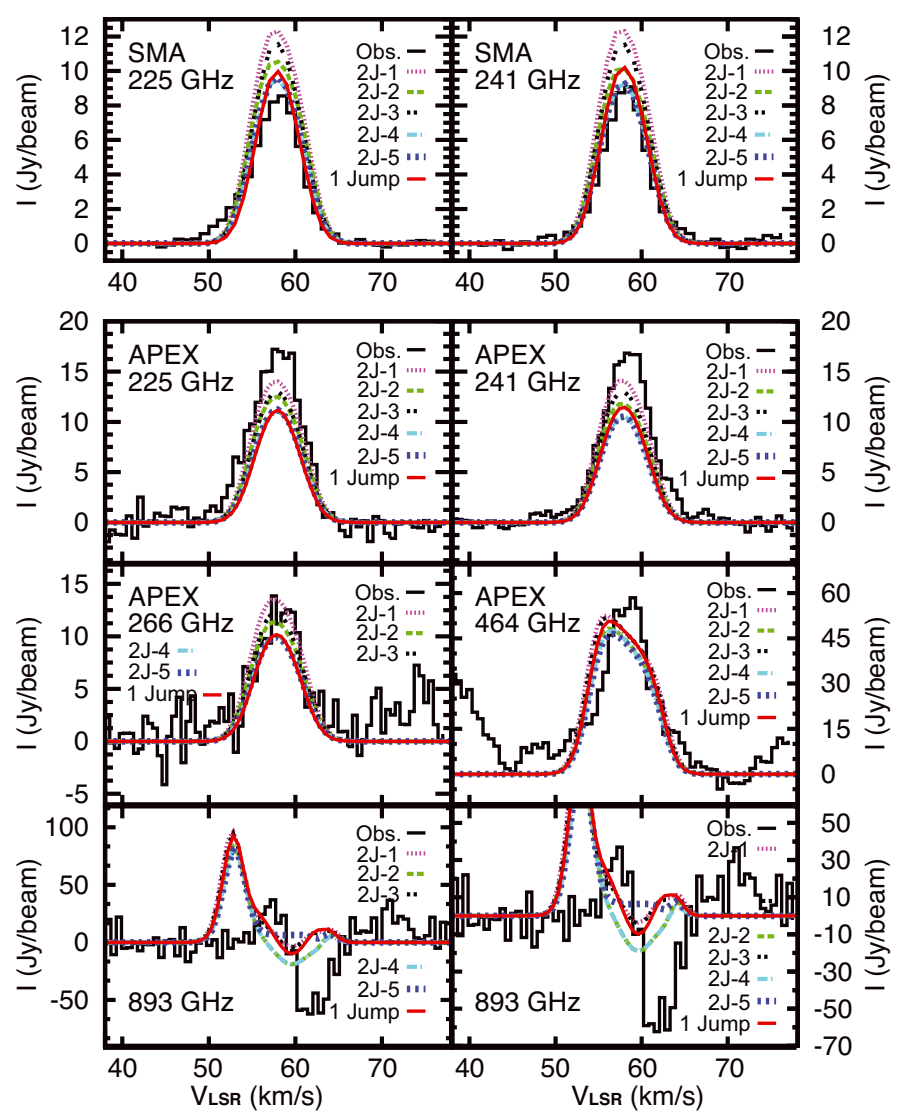

Fig. 9. Comparison of the observed spectra and those simulated with different models. The red lines are the spectra of the original one-jump model.

As a consequence, one cannot increase the inner HDO abundance too much, even if the effect on the APEX lines is almost unnoticeable.

\subsection{Modified physical profiles}

In the previous sections, we have seen that the observed spectra are not perfectly reproduced using the Rolffs physical profile, even with a two-jump model. This is also the case for some HCN lines (see Fig. 5 in Rolffs et al. 2011). We now investigate how the model can be improved by modifying the physical profiles from the Rolffs model. We first investigate where the HDO emission and absorption take place by means of a shell analysis and then test the effect of modfying the physical profiles of the Rolffs model. 

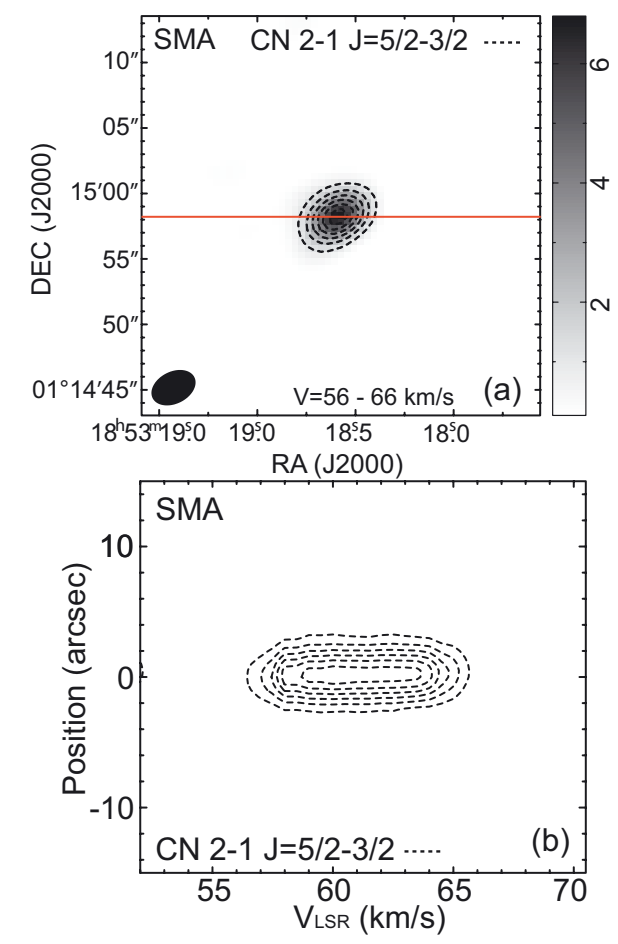

Fig. 10. Integrated moment 0 map of $\mathrm{CN} 2-1 J=5 / 2-3 / 2 F=$ $7 / 2-5 / 2$ line (blended with the $F=5 / 2-3 / 2$ and $F=3 / 2-1 / 2$ lines) overlaid with the dust continuum map a). The red line indicates the cut for the position-velocity $(P-V)$ diagram. The $P-V$ diagram of the $\mathrm{CN}$ line b). Both are observed with SMA. The contours in a) are -0.67 , $-1.34,-2.01,-2.68,-3.35$, and -4.02 and in b) they are $-0.97,-1.94$, $-2.91,-3.88,-4.86$, and -5.83 . The $P-V$ diagram is cut at the position of the observed center and position angle of 0 .

\subsubsection{Shell analysis and modified density/temperature profiles}

To analyze the discrepancy between the best-fit model and the observed data, we performed a shell analysis by sequentially turning the abundance of HDO in the innermost shells to 0 and then simulating new spectra (Fig. A.1). The results of this study are shown in Appendix A.1. We find that the emission of all transitions (even the $893 \mathrm{GHz}$ line) originates in the inner region, whereas the absorption originates in the outer region. This explains why it is impossible to improve the line profile of the $893 \mathrm{GHz}$ within the frame of a jump model. Improvement can therefore only be achieved by modifying the physical profiles (density, temperature, velocity) of the source. In Appendices A.2 and A.3, we show that modifications of the density and temperature profiles are unable to improve the quality of the fit. In the next section, we investigate the effect of modifying the velocity field.

\subsubsection{Modified velocity profile}

\section{- Modified infall velocity field}

So far, we used the velocity profile provided by Rolffs et al. (2011) for all modeling. This is a modified free-fall velocity field. However, the line shape in the $893 \mathrm{GHz}$ spectra cannot be simply reproduced by our present model, which might imply that there is an additional velocity field in the core.

Figure 10 shows the moment- 0 map overlaid with the dust continuum map (a) and the position-velocity diagram (b) of the CN 2-1 absorption line observed with the SMA simultaneously

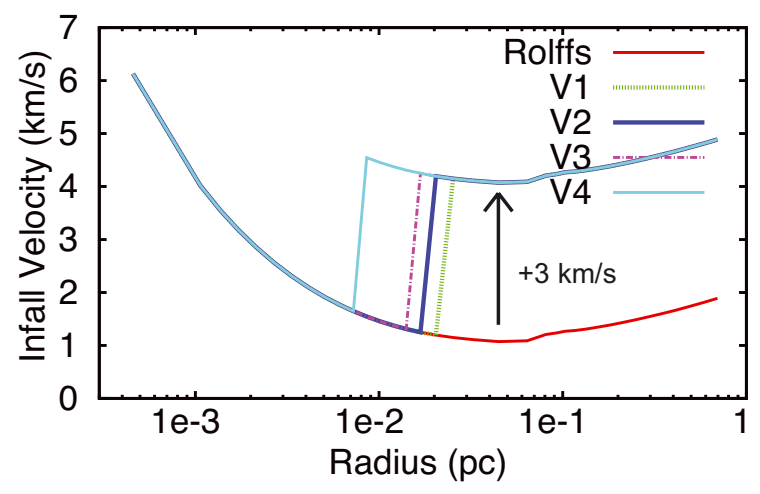

Fig. 11. Comparison of the velocity profiles of the four different modified models (green, blue, purple, and light-blue) and the one of the Rolffs original model (red).

to the HDO lines. Clearly, the distribution of the $\mathrm{CN}$ absorption is consistent with that of the dust continuum, as in the case of HDO. Moreover, the central velocity of the positionvelocity diagram of the $\mathrm{CN}$ absorption is about $61 \mathrm{~km} \mathrm{~s}^{-1}$, not $58 \mathrm{~km} \mathrm{~s}^{-1}$. This redshifted absorption has already been found for many molecules, for instance $\mathrm{NH}_{3}, \mathrm{CO},{ }^{13} \mathrm{CO}$, and $\mathrm{HCO}^{+}$ (Heaton et al. 1989; Gómez et al. 2000; Wyrowski et al. 2012). Thus, it is possible that the gas that produces the absorption feature in the HDO $893 \mathrm{GHz}$ spectra has a similar velocity distribution. Therefore we tried to modify the velocity field by adding a $3 \mathrm{~km} \mathrm{~s}^{-1}$ difference from the outer region to the inner region of the core (Fig. 11). The results are shown in Fig. 12. The spectra are simulated with the fixed fractional abundances $6 \times 10^{-8}$ $\left(\mathrm{X}_{\mathrm{in}}^{\mathrm{HDO}}\right)$ and $5 \times 10^{-12}\left(\mathrm{X}_{\mathrm{out}}^{\mathrm{HDO}}\right)$ (best-fit model). The additional velocity $\left(3 \mathrm{~km} \mathrm{~s}^{-1}\right)$ widens the emission of the optically thin lines $(225,241$, and $266 \mathrm{GHz})$ somewhat, which enables a better fit of the line-width of the SMA lines. The opacities of the HDO 464 and $893 \mathrm{GHz}$ lines are reduced and the $464 \mathrm{GHz}$ line becomes optically thinner in models V3 and V4 $(\tau=1.3$ and 0.8 ). Moreover, the peak positions of the absorption in the simulated $893 \mathrm{GHz}$ spectra gradually approach those of the observed $893 \mathrm{GHz}$ spectra. Therefore, carefully modifying the velocity field might be a good way to improve the fits. However, the detailed velocity profile around the boundary region of the core cannot be constrained from our data now. To obtain better modeling results, it is necessary to observing the HDO line map at $893 \mathrm{GHz}$ with high angular resolution. In addition, this result also suggests that studying the HDO spectrum at $893 \mathrm{GHz}$ can help us to understand the velocity field of the core.

- Modified turbulence velocity width

Because the line profiles are also affected by gas motions, the turbulence is also responsible for the line emission/absorption widths. The assumption that turbulence varies over the core has already been used for analyzing the spectra lines toward several massive sources and brought nicely fitting results (Herpin et al. 2009). Our previous study shows that the width of all simulated 893 spectra is obviously much wider than that of the observed spectrum, while the widths of the high-excitation lines are well fitted. Thus, we modified the turbulent velocity field by changing the parameter $\mathrm{db}$ in the model. In the Fig. 13a, the distribution of the populations of the upper level of highexcitation lines $\left(3_{1,2}, 2_{2,0}\right.$, and $\left.2_{2,1}\right)$ is different from those of the ground-transition lines $\left(1_{1,1}\right.$ and $\left.1_{0,1}\right)$. The peaks of the highexcitation line populations are mainly located in the layer 15-16 $\left(1.4 \times 10^{-2}-1.7 \times 10^{-2} \mathrm{pc}\right)$ and those of the ground-transition lines are placed in the layer $21-24\left(6.4 \times 10^{-2}-1.0 \times 10^{-1} \mathrm{pc}\right)$. 

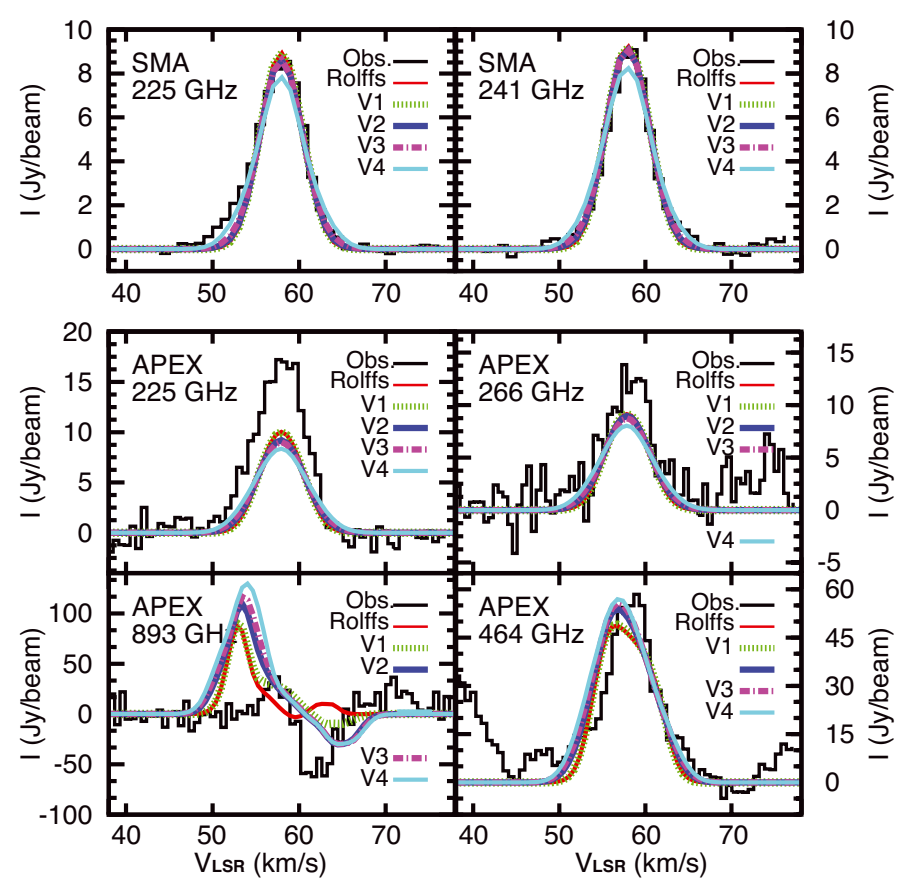

Fig. 12. Comparison of the observed and modeled spectra using the velocity profiles V1-V4 (Fig. 11) and the same HDO fractional abundance as in the best fit. The red lines are the spectra modeled with the bestfit model using the original Rolffs physical profile. The observed and fitting spectra at $241 \mathrm{GHz}$ are very similar to the those at $225 \mathrm{GHz}$; therefore, only the spectra at $225 \mathrm{GHz}$ are plotted here.

We used these considerations for elaborating our new db settings with the aim to narrow down the width of the simulated 893 spectra without significantly affecting the width of the highexcitation lines. Figure 13b illustrates our different db settings. The HDO fractional abundances in the model $\mathrm{db} 1-\mathrm{db} 4$ are fixed with the values of the best-fit model: $\mathrm{X}_{\text {in }}^{\mathrm{HDO}}=6 \times 10^{-8}$ and $\mathrm{X}_{\mathrm{out}}^{\mathrm{HDO}}=5 \times 10^{-12}$.

Comparing the spectra at $893 \mathrm{GHz}$ with those of the previous best-fit model (Fig. 14), we find that the line profile has significantly improved much in $\mathrm{db} 2-\mathrm{db} 4$. The width of the absorption part is narrower than before and more similar to the observed one. Moreover, we have better fits compared to previous spectra at $266 \mathrm{GHz}$ and the opacity is reduced in the $464 \mathrm{GHz}$ spectra. In addition, the $\chi_{\text {red }}^{2}$ model db2 is 5.5 , which is better than that of the Rolffs original model (6.0), showing that the fits can be improved by modifying the turbulent velocity field. We also performed $\chi^{2}$ analyses for the same range of $\mathrm{X}_{\text {in }}^{\mathrm{HDO}}$ and $\mathrm{X}_{\mathrm{out}}^{\mathrm{HDO}}$ in Sect. 4.2 based on model db2. The fractional abundances of the new best-fit in this test are still the same as in our previous results $\left(\mathrm{X}_{\text {in }}^{\mathrm{HDO}}=6 \times 10^{-8}\right.$ and $\left.\mathrm{X}_{\mathrm{out}}^{\mathrm{HDO}}=5 \times 10^{-12}\right)$. This implies that the turbulence velocity is not constant throughout the envelope of G34.26+0.15. However, the widths at 225 and $241 \mathrm{GHz}$ are narrower than the previous and observed ones, suggesting that the $\mathrm{db}$ in the inner region of the core is not wide enough in the models. Here again, high angular resolution data at $893 \mathrm{GHz}$ would be very helpful.

- Composition of the turbulent and infall velocity field effects

Because modifying the infall velocity field can help to move the peak of the absorption at $893 \mathrm{GHz}$ (model V1), and modifying the turbulence velocity field can narrow this absorption, we ran models with modified velocity (V1 and db2). The result is shown in Fig. 15. Obviously, we can now reproduce part of

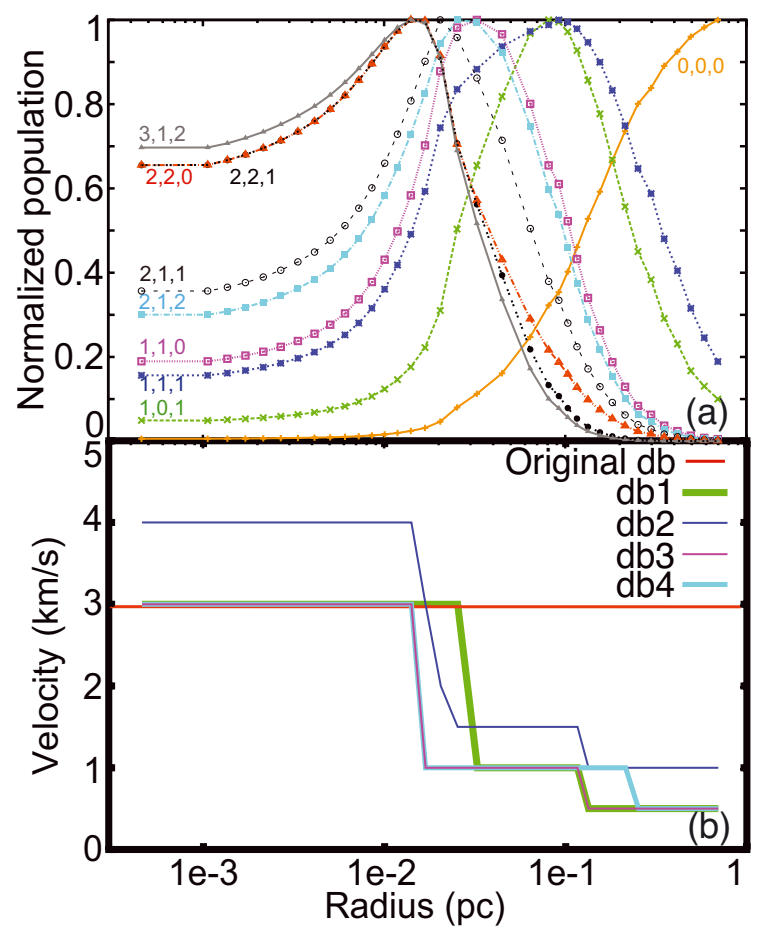

Fig. 13. HDO normalized population of the selected levels of the Rolffs original model a) and comparison of the turbulent velocity fields of the four models $(\mathrm{db} 1-\mathrm{db} 4)$ and the turbulent velocity fields of the original model (red) b).

the emission and all the absorption with better width and proper position, which allows us to study the fractional abundance in the outer region of the core. On the other hand, as expected, the emission at $893 \mathrm{GHz}$ is not reproduced properly, because it originates from the inner region of the core $(T>100 \mathrm{~K})$ and the model V1 only changes the velocity field in the outer region of the core $(T<100 \mathrm{~K})$. Additional constraints in this direction will hopefully come from interferometeric data.

In conclusion, we have shown that it is possible to improve the original best-fit model by modifying the velocity field of the source. This improvement is obtained while keeping the fractional abundance of HDO to the value we derived with the original velocity setup of Rolffs et al. (2011). This study confirms the validity of the fractional abundance of HDO derived from the best-fit model in Sect. 4.3. Further steps toward constraining the velocity field and therefore a better HDO model will need high angular resolution observations of, e.g., the HDO $893 \mathrm{GHz}$ line.

\section{3. $\mathrm{HDO} / \mathrm{H}_{2} \mathrm{O}$ ratio}

$\mathrm{H}_{2}^{18} \mathrm{O}$ is always used to trace $\mathrm{H}_{2} \mathrm{O}$, because $\mathrm{H}_{2} \mathrm{O}$ is easily optically thick. Here we compare the emission and absorption of the two para- $\mathrm{H}_{2}^{18} \mathrm{O}$ lines $3_{13}-2_{20}$ and $1_{11}-0_{00}$ (Gensheimer et al. 1996; Wyrowski et al. 2010) with our HDO results. Because the analysis of the HDO lines suggests that high-excitation lines can trace and constrain the inner fractional abundance quite well (Parise et al. 2005b; Liu et al. 2011), we can also analyze the high-excitation $\mathrm{H}_{2}^{18} \mathrm{O} 3_{13}-2_{20}$ line $\left(E_{\mathrm{u}}=204.7 \mathrm{~K}\right)($ Gensheimer et al. 1996) to study the $\mathrm{H}_{2}^{18} \mathrm{O}$ fractional abundance in the inner region of the core. We reproduced the reported integrated flux and line width $\left(20.5 \mathrm{~K}\right.$ and $8.4 \mathrm{~km} \mathrm{~s}^{-1}$ listed in Table 2 in Gensheimer et al. 1996) of this $\mathrm{H}_{2}^{18} \mathrm{O}$ line with RATRAN using a one-jump model based on the Rolffs original profiles. The 

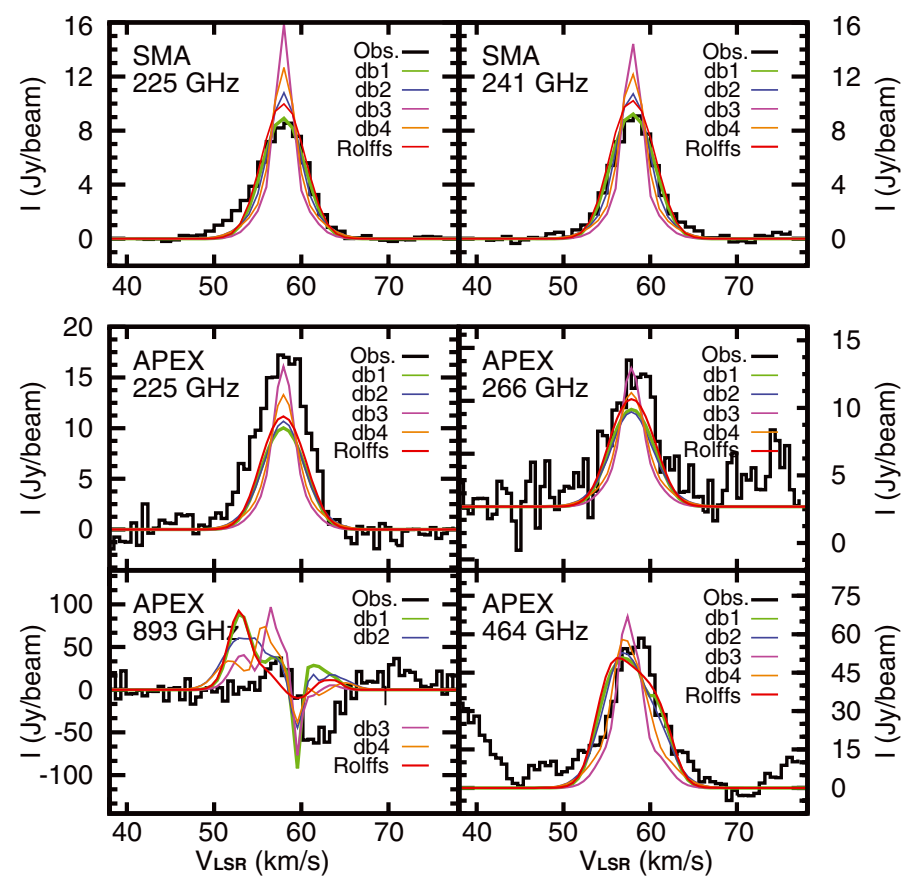

Fig. 14. Same as Fig. 12, but for the models with different settings of the turbulent velocity field $(\mathrm{db} 1-\mathrm{db} 4)$.

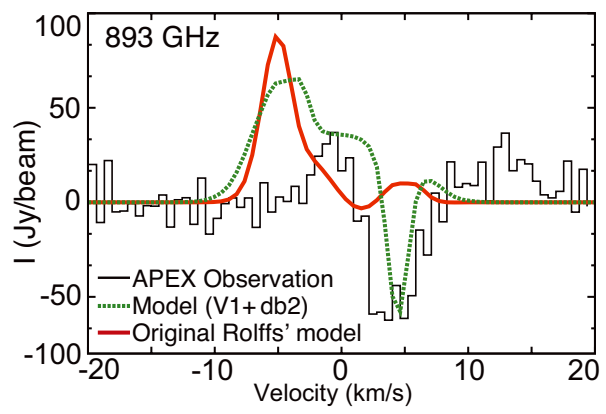

Fig. 15. Comparison of the observed spectrum, the one of the best-fit model simulated with Rolffs' original physical profile, and the one reproduced with the modified turbulent and infall velocity fields (V1 and $\mathrm{db} 2$ ). The fractional abundance adopted here is the one of the best-fit model.

resulting fractional abundance of the para- $\mathrm{H}_{2}^{18} \mathrm{O}$ in the inner region of the core is $(1.0 \pm 0.7) \times 10^{-7}(3 \sigma)$. Thus, the $\mathrm{HDO} / \mathrm{H}_{2} \mathrm{O}$ is $3.0 \times 10^{-4}$ with the assumption that the standard ortho/para ratio is 3 and the ${ }^{18} \mathrm{O} /{ }^{16} \mathrm{O}$ ratio is 500 . This ratio is consistent with the estimate of $1.1 \times 10^{-4}$ with an uncertainty of a factor of 4 (Gensheimer et al. 1996). Here, the adopted db for this high-excitation $\mathrm{H}_{2}^{18} \mathrm{O}$ line is $4.5 \mathrm{~km} \mathrm{~s}^{-1}$ instead of $3 \mathrm{~km} \mathrm{~s}^{-1}$, because the width of this $\mathrm{H}_{2}^{18} \mathrm{O}$ line is wider than that of the highexcitation HDO lines ( $8.4 \mathrm{~km} \mathrm{~s}^{-1}$ for the $\mathrm{H}_{2}^{18} \mathrm{O}$ line compared to $\sim 6-7 \mathrm{~km} \mathrm{~s}^{-1}$ for the HDO lines). The different properties of the velocity field of the high-excitation $\mathrm{H}_{2}^{18} \mathrm{O}$ line may indicate that some part of the emission does not come from the quiescent envelope and the high-excitation HDO lines may not probe exactly the same gas. Therefore, the derived $\mathrm{D} / \mathrm{H}$ ratio of water in the inner region here should be a lower limit.

Figure 16 displays the two spectra of the ground-state $1_{1,1}-0_{0,0}$ transition of $\mathrm{HDO}$ and $\mathrm{H}_{2}^{18} \mathrm{O}$. The line shapes are very similar, suggesting they may originate from the same gas. Under the two assumptions that the excitation temperature of the line is negligible with respect to the temperature of the
Table 6. Line parameters and continuum temperature corresponding to the frequency of each line.

\begin{tabular}{cccclll}
\hline \hline & $\begin{array}{c}\text { Freq. } \\
\mathrm{GHz}\end{array}$ & $\begin{array}{c}T_{\text {peak }}{ }^{a} \\
\mathrm{~K}\end{array}$ & $\begin{array}{c}T_{\text {cont }^{a b}} \\
\mathrm{~K}\end{array}$ & $\begin{array}{l}\Delta v \\
\mathrm{~km} \mathrm{~s}^{-1}\end{array}$ & $\tau$ & $\begin{array}{c}V_{\text {LSR }} \\
\mathrm{km} \mathrm{s}^{-1}\end{array}$ \\
\hline $\mathrm{HDO}$ & 893.639 & $-1.89 \pm 0.37$ & 13.0 & $3.65 \pm 0.24$ & 0.17 & $61.9 \pm 0.13$ \\
$\mathrm{H}_{2}^{18} \mathrm{O}$ & 1101.698 & $-1.20 \pm 0.07$ & 6.1 & $3.51 \pm 0.06$ & 0.22 & $61.3 \pm 0.03$ \\
\hline
\end{tabular}

Notes. The parameters errors are obtained from the Gaussian fit. ${ }^{(a)} T_{\mathrm{mb}}$ scale. ${ }^{(b)}$ continuum temperature.

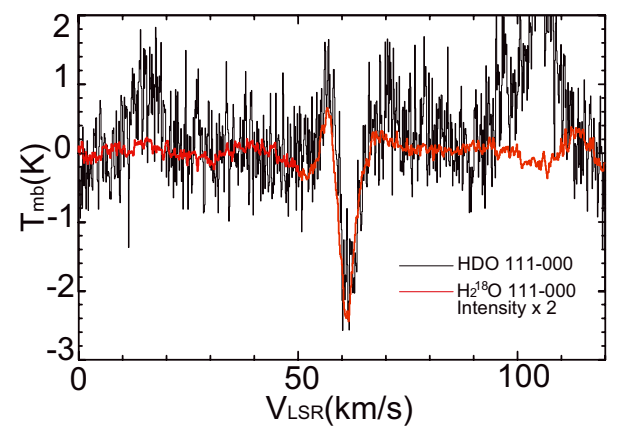

Fig. 16. Comparison of the $\mathrm{HDO}$ at $893 \mathrm{GHz}$ (black) with $\mathrm{H}_{2}^{18} \mathrm{O} 1_{11}-0_{00}$ (red) spectra. The $\mathrm{H}_{2}^{18} \mathrm{O}$ data are taken from Wyrowski et al. (2010).

background continuum source and that only the ground state of the molecule is populated, the total column density of the absorbing $\mathrm{HDO} / \mathrm{H}_{2}^{18} \mathrm{O}$ can then be computed from (Comito et al. 2003; Tarchi et al. 2004)

$N_{\mathrm{tot}}=\frac{8 \pi v^{3}}{A_{u l} c^{3}} \frac{g_{l}}{g_{u}} \tau \Delta v$,

where $\Delta v$ is the line width and $g_{l}$ and $g_{u}$ are the statistical weights of the lower and upper level. The optical depth of the absorbed line can be determined by

$\tau=-\ln \left(1-\frac{T_{\mathrm{L}}}{T_{\mathrm{C}}}\right)$,

where $T_{\mathrm{L}}$ is the brightness temperature of the line and $T_{\mathrm{C}}$ is the brightness temperature of the continuum. The line parameters from the Gaussian fit and the estimated values are listed in Table 6. The estimated total column densities are $1.8 \times 10^{12} \mathrm{~cm}^{-2}$ for $\mathrm{HDO}$ and $1.8 \times 10^{12} \mathrm{~cm}^{-2}$ for para- $\mathrm{H}_{2}^{18} \mathrm{O}$. Because the profiles of these two lines are very similar to each other, we assumed that both of them are originating from the same gas. Therefore, the $[\mathrm{HDO}] /\left[\mathrm{H}_{2} \mathrm{O}\right]$ ratio is $\sim 4.9 \times 10^{-4}$, assuming that the standard ortho/para ratio is 3 and the ${ }^{18} \mathrm{O} /{ }^{16} \mathrm{O}$ ratio is 500 . However, the abundance of para- $\mathrm{H}_{2}^{18} \mathrm{O}$ might be underestimated here, because the emitted area of the continuum at $1101 \mathrm{GHz}$ might be larger than that of the $\mathrm{H}_{2}^{18} \mathrm{O}$ due to the large Herschel beam. Assuming that the dust emissivity spectral index $\beta$ is 2 as for the interstellar medium (Draine \& Lee 1984; Schnee et al. 2010), the derived continuum flux at $1101 \mathrm{GHz}$ of the core seen with APEX will be $2.8 \mathrm{~K}$ in the Herschel beam and the estimated total column $\mathrm{H}_{2}^{18} \mathrm{O}$ density becomes $4.7 \times 10^{12} \mathrm{~cm}^{-2}$. Then the obtained $\mathrm{D} / \mathrm{H}$ ratio of water is $\sim 1.9 \times 10^{-4}$, which is close to what we obtained for the inner region of the core. We note that this value is uncertain, as the absorption takes place on top of emission in both cases, which we have ignored here. 
Table 7. Comparison of HDO fractional abundance between different sources.

\begin{tabular}{|c|c|c|c|c|c|c|}
\hline \multirow[t]{2}{*}{ Source } & \multicolumn{2}{|c|}{$\mathrm{X}^{\mathrm{HDO}}$ (best fit) } & \multicolumn{2}{|r|}{$\frac{\mathrm{HDO}}{\mathrm{H}_{2} \mathrm{O}}$} & \multirow[t]{2}{*}{$\frac{\mathrm{HDCO}}{\mathrm{H}_{2} \mathrm{CO}}$} & \multirow[t]{2}{*}{$\frac{\mathrm{DCN}}{\mathrm{HCN}}$} \\
\hline & Inner & outer & Inner & outer & & \\
\hline & \multicolumn{5}{|c|}{ High-mass hot cores } & \\
\hline $\begin{array}{l}\text { G34.26+0.15 } \\
\text { AFGL } 2591 \\
\text { W33A } \\
\text { Orion KL }\end{array}$ & $\begin{array}{c}6 \times 10^{-8 a} \\
1 \times 10^{-7 b} \\
2 \times 10^{-7 b} \\
\ldots \\
\end{array}$ & $\begin{array}{c}5 \times 10^{-12 a} \\
4 \times 10^{-9 b} \\
1 \times 10^{-8 b} \\
\quad \ldots \\
\end{array}$ & $\begin{array}{l}3 \times 10^{-4 a} \\
5 \times 10^{-4 b}\end{array}$ & $\begin{array}{l}1.9 \times 10^{-4 a}-4.9 \times 10^{-4 a} \\
0.4-4 \times 10^{-3 b} \\
7 \times 10^{-3 b} \\
2 \times 10^{-2 c}\end{array}$ & $\begin{array}{c}0.01^{h} \\
\ldots \\
\ldots \\
0.14^{d}\end{array}$ & $\begin{array}{c}0.001^{h} \\
\ldots \\
\ldots \\
0.005-0.02^{k}\end{array}$ \\
\hline Orion KL & \multicolumn{5}{|c|}{ Low-mass protostars } & \\
\hline $\begin{array}{l}\text { NGC } 1333 \text { IRAS2A } \\
\text { IRAS 16293-2422 }\end{array}$ & $\begin{array}{l}8 \times 10^{-8 e} \\
1 \times 10^{-7 g} \\
1.7 \times 10^{-7 i} \\
\end{array}$ & $\begin{array}{c}7 \times 10^{-10 e} \\
1.5 \times 10^{-10 g} \\
8 \times 10^{-11 i} \\
\end{array}$ & $\begin{array}{l}21 \times 10^{-2 e} \\
3 \times 10^{-2 g} \\
3.4 \times 10^{-2 i}\end{array}$ & $\begin{array}{c}7_{-6}^{+11} \times 10^{-2 e} \\
\leq 2 \times 10^{-3}(3 \sigma)^{g} \\
5 \times 10^{-3 i}\end{array}$ & $\begin{array}{c}0.17_{-0.08}^{+0.12 f} \\
0.15 \pm 0.07^{f}\end{array}$ & $0.013^{m}$ \\
\hline
\end{tabular}

Notes. ${ }^{(a)}$ This work. ${ }^{(b)}$ van der Tak et al. (2006); ${ }^{(c)}$ Bergin et al. $(2010) ;{ }^{(d)}$ Turner (1990); ${ }^{(e)}$ Liu et al. $(2011) ;(f)$ Parise et al. $(2006) ;(g)$ Parise et al. (2005a); ${ }^{(h)}$ Roberts \& Millar (2007); ${ }^{(i)}$ Coutens et al. (2012); ${ }^{(k)}$ Mangum et al. (1991); ${ }^{(m)}$ van Dishoeck et al. (1995).

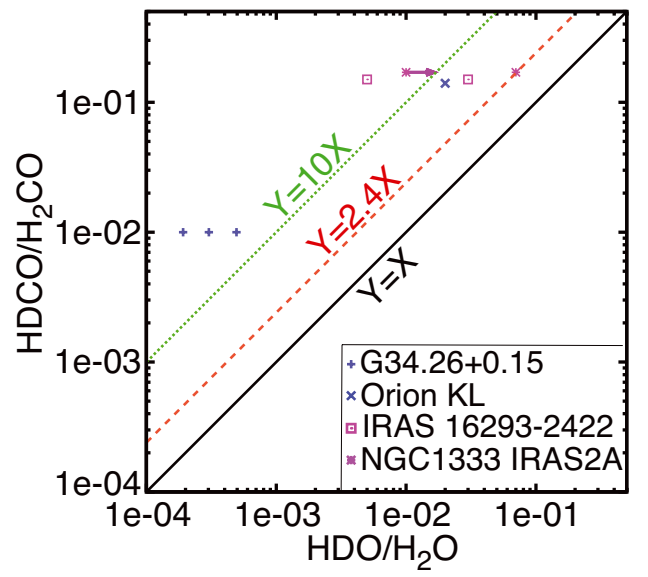

Fig. 17. Diagram of $\mathrm{HDO} / \mathrm{H}_{2} \mathrm{O}$ vs. $\mathrm{HDCO} / \mathrm{H}_{2} \mathrm{CO}$. Data are taken from Table 7.

The comparison of the HDO fractional abundances between different sources is shown in Table 7. The water deuterium fractionation toward the two low-mass protostars, NGC1333 IRAS 2A and IRAS 16293-2422, have been studied with several HDO transitions (Liu et al. 2011; Parise et al. 2005a; Coutens et al. 2012). Most transitions were also used in this work.

The abundance of HDO jumps by more than four orders of magnitude at the $3 \sigma$ confidence level in G34.26+0.15. Similarly to this work, jump models are widely used to analyze the HDO and $\mathrm{H}_{2} \mathrm{O}$ spectra of other high- and low-mass sources in Table 7, except for Orion KL. Ices are therefore also shown to evaporate from the grains in the inner part of the envelope in protostars of different masses. However, the $\mathrm{D} / \mathrm{H}$ ratios of water in G34.26+0.15 is much lower than in low-mass protostars and similar to that in AFGL 2591 and other high-mass sources, such as Ori-IRc2, G31.41+0.31, and G10.47+0.03A (van der Tak et al. 2006; Gensheimer et al. 1996). In addition, the D/H ratios of formaldehyde and $\mathrm{HCN}$ in G34.26+0.15 are also lower than those in low-mass protostars. This can be explained if the dense and cold pre-collapse phase only lasts a short time and less CO freezes onto grain mantles. This hypothesis has been confirmed in the high-mass source AFGL 2591 (van der Tak et al. 2006).

Figure 17 shows the diagram of $\mathrm{HDO} / \mathrm{H}_{2} \mathrm{O}$ vs. $\mathrm{HDCO} / \mathrm{H}_{2} \mathrm{CO}$ and the values are taken from Table 7 . It is found that the fractionation of water is at least $\sim 2.4$ times lower than that of formaldehyde. This under-fractionation of water is found to be the most extreme for $\mathrm{G} 34.26+0.15$. One possible explanation is that if two molecules are mainly formed on the grain surface, the different deuterium fractionation can be due to the different pathways of each ice formation in translucent clouds: using a very simple chemical network on the grains, Cazaux et al. (2011) showed that the deuteration of formaldehyde is sensitive to the gas $\mathrm{D} / \mathrm{H}$ ratio during the cloud collapse, while the deuteration of water depends on the dust temperature when ices form. More realistic models including water formation routes involving $\mathrm{O}_{2}$ and $\mathrm{O}_{3}$ also predict a higher fractionation of formaldehyde than water (Du et al. in prep.). However, recent studies showed that formaldehyde can also be formed in the gas phase from photodissociation reactions of grain species (Roueff et al. 2006). Formaldehyde can also efficiently be fractionated in the gas phase up to warm temperatures $(\sim 70 \mathrm{~K})$, through the $\mathrm{CH}_{2} \mathrm{D}^{+}$ route (Turner 2001; Roueff et al. 2007; Parise et al. 2009). It is therefore likely that the $\mathrm{HDCO} / \mathrm{H}_{2} \mathrm{CO}$ ratio observed in the gas is not directly the result of grain chemistry. A better comparison would be with methanol fractionation, which is generally thought to form essentially on the grains.

Confirming the observed trend of under-fractionation of water would require observationally characterizing the deuterium fractionation (for both water and methanol) toward a bigger sample of sources, spanning a large mass interval. This underdeuteration of water with respect to other species is an important feature, which should be reproduced by astrochemical models aiming at explaining deuterium fractionation.

\section{Conclusion}

We presented five HDO transitions observed with APEX and two HDO high-excitation transitions observed with SMA toward the high-mass protostar, G34.26+0.15. With the $1 \mathrm{D}$ radiative transfer code RATRAN, and the physical profiles obtained from Rolffs et al. (2011), we derived the HDO fractional abundances relative to $\mathrm{H}_{2}$ in the inner and outer region of the core to be $\mathrm{X}_{\text {in }}^{\mathrm{HDO}}=6 \times 10^{-8}(T>100 \mathrm{~K})$ and $\mathrm{X}_{\text {out }}^{\mathrm{HDO}}=5 \times 10^{-12}$ $(T \leq 100 \mathrm{~K})$. This result shows that the HDO abundance is enriched in the inner region because of the sublimation of the ice in the same way as for other studied low- and high-mass sources, such as NGC1333 IRAS 2A, IRAS 16293-2422, and AFGL 2591 (Liu et al. 2011; Parise et al. 2005a; Coutens et al. 2012; van der Tak et al. 2006). 
Although the abundance can be constrained, the HDO line profiles are not well reproduced, especially for the ground-state lines. We found that the fitted emission can be improved with two-jump models, suggesting the enhancement of the fractional abundance in the hot core. Modifying the physical profile, we showed that the velocity field is responsible for the HDO line profile at $893 \mathrm{GHz}$. Higher angular resolution observations of the HDO $893 \mathrm{GHz}$ line are needed to constrain the velocity profiles of deuterated water in $\mathrm{G} 34.26+0.15$.

The $\mathrm{H}_{2} \mathrm{O}$ abundance is estimated from one high-excitation and one ground transition para- $\mathrm{H}^{18} \mathrm{O}$ line (Gensheimer et al. 1996; Wyrowski et al. 2010). The $\mathrm{D} / \mathrm{H}$ ratios of water are $3.0 \times$ $10^{-4}$ in the inner region and $(1.9-4.9) \times 10^{-4}$ in the outer region of the core. The deduced $\mathrm{HDO} / \mathrm{H}_{2} \mathrm{O}$ ratios in $\mathrm{G} 34.26+0.15$ are similar to those in other high-mass sources, such as AFGL 2591 (van der Tak et al. 2006), and are much lower than in low-mass star-forming regions, suggesting the possibility that the dense and cold pre-collapse phase is short for high-mass stars. Water is also found to be less fractionated than other molecules such as formaldehyde and methanol. This confirms this characteristic, which was observed previously in other sources, and provides a challenge for chemical models of deuterium fractionation on grain surfaces.

Acknowledgements. The authors warmly thank the anonymous referee and $\mathrm{M}$ Walmsley for very constructive comments and suggestions, which greatly improved this paper. We are grateful to R. Rolffs for many fruitful discussions as well as to F. Herpin for some interesting ideas. We also would like to thank M. Hogerheijde and F. van der Tak for their help in dealing with RATRAN. The authors are grateful to the WISH team for providing access to the $\mathrm{H}_{2} \mathrm{O}$ data. F.-C. Liu and B. Parise are funded by the German Deutsche Forschungsgemeinschaft, DFG Emmy Noether project number PA1692/1-1.

\section{References}

Andersson, M., \& Garay, G. 1986, A\&A, 167, L1

Avalos, M., Lizano, S., Rodríguez, L. F., Franco-Hernández, R., \& Moran, J. M. 2006, ApJ, 641, 406

Bergin, E. A., Melnick, G. J., Stauffer, J. R., et al. 2000, ApJ, 539, L129

Bergin, E. A., Phillips, T. G., Comito, C., et al. 2010, A\&A, 521, L20

Carral, P., \& Welch, W. J. 1992, ApJ, 385, 244

Cazaux, S., Caselli, P., \& Spaans, M. 2011, ApJ, 741, L34

Chavarría, L., Herpin, F., Jacq, T., et al. 2010, A\&A, 521, L37

Chini, R., Kruegel, E., \& Wargau, W. 1987, A\&A, 181, 378

Comito, C., Schilke, P., Gérin, M., et al. 2003, A\&A, 402, 635

Comito, C., Schilke, P., Rolffs, R., et al. 2010, A\&A, 521, L38

Condon, J. J. 1997, PASP, 109, 166

Coutens, A., Vastel, C., Caux, E., et al. 2012, A\&A, 539, A132

Cuppen, H. M., Ioppolo, S., Romanzin, C., \& Linnartz, H. 2010, Phys. Chem. Chem. Phys. (Incorporating Faraday Transactions), 12, 12077

Draine, B. T., \& Lee, H. M. 1984, ApJ, 285, 89

Faure, A., Wiesenfeld, L., Scribano, Y., \& Ceccarelli, C. 2012, MNRAS, 420, 699

Fey, A. L., Claussen, M. J., Gaume, R. A., Nedoluha, G. E., \& Johnston, K. J. 1992, AJ, 103, 234

Gaume, R. A., Fey, A. L., \& Claussen, M. J. 1994, ApJ, 432, 648
Gensheimer, P. D., Mauersberger, R., \& Wilson, T. L. 1996, A\&A, 314, 281

Goldsmith, P. F., \& Langer, W. D. 1999, ApJ, 517, 209

Gómez, Y., Rodríguez-Rico, C. A., Rodríguez, L. F., \& Garay, G. 2000, Rev.

Mex. Astron. Astronfis, 36, 161

Green, S. 1986, ApJ, 309, 331

Green, S. 1994, ApJ, 434, 188

Green, S., \& Chapman, S. 1978, ApJS, 37, 169

Hatchell, J., Millar, T. J., \& Rodgers, S. D. 1998a, A\&A, 332, 695

Hatchell, J., Thompson, M. A., Millar, T. J., \& MacDonald, G. H. 1998b, A\&AS, 133,29

Heaton, B. D., Little, L. T., \& Bishop, I. S. 1989, A\&A, 213, 148

Herpin, F., Marseille, M., Wakelam, V., Bontemps, S., \& Lis, D. C. 2009, A\&A, 504,853

Hogerheijde, M. R., \& van der Tak, F. F. S. 2000, A\&A, 362, 697

Jacq, T., Walmsley, C. M., Henkel, C., et al. 1990, A\&A, 228, 447

Kristensen, L. E., van Dishoeck, E. F., van Kempen, T. A., et al. 2010a, A\&A, 516, A57

Kristensen, L. E., Visser, R., van Dishoeck, E. F., et al. 2010b, A\&A, 521, L30

Kuchar, T. A., \& Bania, T. M. 1994, ApJ, 436, 117

Lampton, M., Margon, B., \& Bowyer, S. 1976, ApJ, 208, 177

Linsky, J. L. 2003, Space Sci. Rev., 106, 49

Liu, F.-C., Parise, B., Kristensen, L., et al. 2011, A\&A, 527, A19

Loinard, L., Castets, A., Ceccarelli, C., Caux, E., \& Tielens, A. G. G. M. 2001 ApJ, 552, 163

Mangum, J. G., Plambeck, R. L., \& Wootten, A. 1991, ApJ, 369, 169

Mookerjea, B., Casper, E., Mundy, L. G., \& Looney, L. W. 2007, ApJ, 659, 447

Nomura, H., \& Millar, T. J. 2004, A\&A, 414, 409

Ossenkopf, V., \& Henning, T. 1994, A\&A, 291, 943

Pardo, J. R., Cernicharo, J., Herpin, F., et al. 2001, ApJ, 562, 799

Parise, B., Castets, A., Herbst, E., et al. 2004, A\&A, 416, 159

Parise, B., Caux, E., Castets, A., et al. 2005a, A\&A, 431, 547

Parise, B., Ceccarelli, C., \& Maret, S. 2005b, A\&A, 441, 171

Parise, B., Ceccarelli, C., Tielens, A. G. G. M., et al. 2006, A\&A, 453, 949

Parise, B., Leurini, S., Schilke, P., et al. 2009, A\&A, 508, 737

Pottage, J. T., Flower, D. R., \& Davis, S. L. 2004, MNRAS, 352, 39

Roberts, H., \& Millar, T. J. 2007, A\&A, 471, 849

Rolffs, R., Schilke, P., Wyrowski, F., et al. 2011, A\&A, 527, A68

Roueff, E., Tiné, S., Coudert, L. H., et al. 2000, A\&A, 354, L63

Roueff, E., Lis, D. C., van der Tak, F. F. S., Gérin, M., \& Goldsmith, P. F. 2005, A\&A, 438, 585

Roueff, E., Dartois, E., Geballe, T. R., \& Gerin, M. 2006, A\&A, 447, 963

Roueff, E., Parise, B., \& Herbst, E. 2007, A\&A, 464, 245

Schnee, S., Enoch, M., Noriega-Crespo, A., et al. 2010, ApJ, 708, 127

Schöier, F. L., van der Tak, F. F. S., van Dishoeck, E. F., \& Black, J. H. 2005, A\&A, 432, 369

Snell, R. L., \& Wootten, H. A. 1977, ApJ, 216, L111

Tarchi, A., Greve, A., Peck, A. B., et al. 2004, MNRAS, 351, 339

Tielens, A. G. G. M., \& Hagen, W. 1982, A\&A, 114, 245

Turner, B. E. 1990, ApJ, 362, L29

Turner, B. E. 2001, ApJS, 136, 579

van Buren, D., Mac Low, M.-M., Wood, D. O. S., \& Churchwell, E. 1990, ApJ, 353,570

van der Tak, F. F. S., Walmsley, C. M., Herpin, F., \& Ceccarelli, C. 2006, A\&A, 447, 1011

van Dishoeck, E., Blake, G., Jansen, D., \& Groesbeck, T. 1995, ApJ, 447, 760

van Dishoeck, E. F., Kristensen, L. E., Benz, A. O., et al. 2011, PASP, 123, 138

Wagner, A. F., \& Graff, M. M. 1987, ApJ, 317, 423

Watt, S., \& Mundy, L. G. 1999, ApJS, 125, 143

Wood, D. O. S., \& Churchwell, E. 1989, ApJS, 69, 831

Wootten, A., Loren, R. B., \& Snell, R. L. 1982, ApJ, 255, 160

Wyrowski, F., van der Tak, F., Herpin, F., et al. 2010, A\&A, 521, L34

Wyrowski, F., Güsten, R., Menten, K. M., Wiesemeyer, H., \& Klein, B. 2012, A\&A, 542, L15 


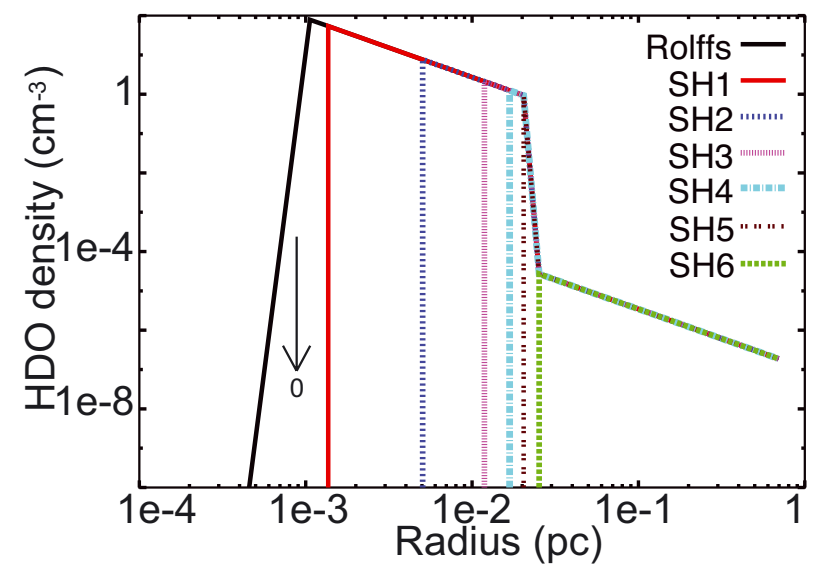

Fig. A.1. Original and modified molecular density profiles. The black line shows the original molecular density profile. Different colors indicate different molecular density profiles of models SH1 to SH6.

\section{Appendix A: Model analysis}

\section{A.1. Shell analysis}

To analyze the discrepancy between the best-fit model based on the Rolffs physical profiles and the observed data for the two ground transitions (464 and $893 \mathrm{GHz}$ ), we first constrained the physical shells responsible for the emission and absorption features. To this end, we simulated new spectra by sequentially turning the abundance of HDO in the innermost shells to 0 (Fig. A.1). The jump around $0.02 \mathrm{pc}$ is at the shell whose temperature is about $100 \mathrm{~K}$ (jump model) The green dashed line represents the model where the HDO abundance is zero in all inner regions of the core (model SH6). Figure A.2 presents the synthetic spectra obtained with these different models (SH1 - SH6). Obviously there is almost no emission in any spectrum with model SH6, suggesting that all emission, including the emission in the $893 \mathrm{GHz}$ spectrum, is produced in the inner region of the core. Therefore, although loweringn the abundance in the inner region can help to reduce the emission in the $893 \mathrm{GHz}$ line, it will also decrease the emission in other high-excitation lines $(225,241$, and $266 \mathrm{GHz})$. On the other hand, the absorption in the $893 \mathrm{GHz}$ spectrum is entirely produced in the outer region of the core and its line-width is wider than the observed one, implying that the assumed turbulent linewidth is wider than the observed one. Thus, we cannot adequately reproduce all spectra with the Rolffs original physical profiles.

\section{A.2. Modified density profile}

From previous test, it was found that the emission in the $893 \mathrm{GHz}$ spectrum cannot be managed by the Rolffs physical profiles, but only by changing the HDO abundance. We therefore modified in the first place the density profile of the Rolffs model. Figure A. 3 compares the density profiles of three different modified models and original the Rolffs model (red line). Here we modified the density power law index (p), keeping the density at $0.1 \mathrm{pc}$ fixed. The indices are 1.1 for model D1 (green), 1.7 for model D2 (dark-blue), and 2 for model D3 (purple). Modifying the density profile has a direct impact on the distribution of the continuum emission. The comparison of the radial profiles of the continuum emission of different models at multi-wavelength bands $(225,241,335$, and $848 \mathrm{GHz})$ is shown in Fig. A.4. Ignoring the misfitting parts within the beam (orange) and considering that free-free emission most likely also significantly
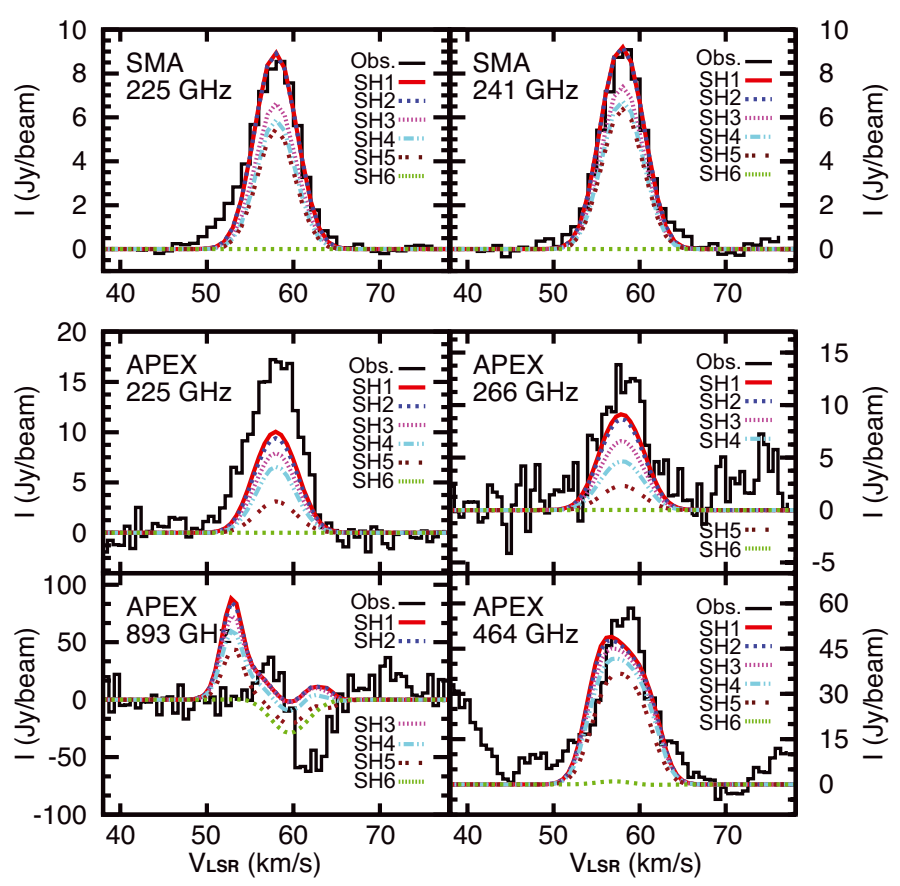

Fig. A.2. Comparison of the observed spectra with the results of 6 different shell models (Fig. A.1). Different colors indicate the simulated lines of models SH1 to SH6. The observed and simulated spectra at $241 \mathrm{GHz}$ are very similar to the spectra at $225 \mathrm{GHz}$; therefore, only the spectra at $225 \mathrm{GHz}$ are plotted here.

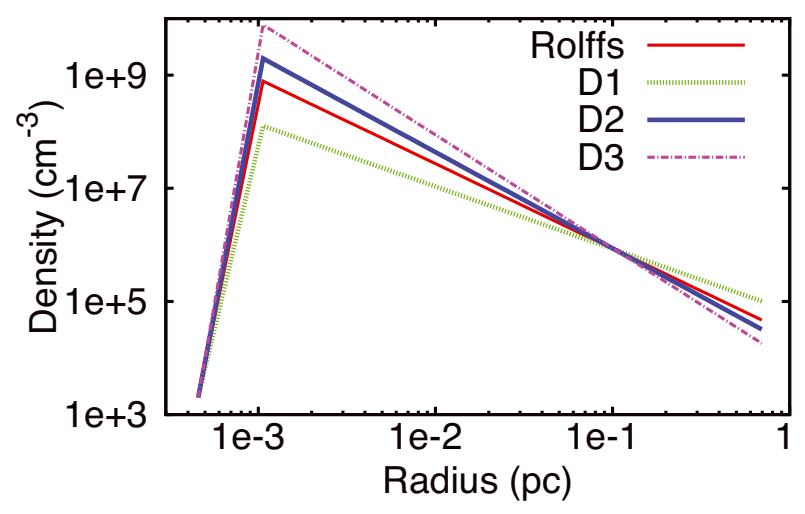

Fig. A.3. Comparison of the density profile of the three different modified models (blue, green, and pink) and original Rolffs' model (red).

contributes to the continuum emission, model D1 and the Rolffs model better reproduce the continuum emission in the 225 and $241 \mathrm{GHz}$ bands than other models. For the 335 and $848 \mathrm{GHz}$ band, the Rolffs model and all modified models are superior to the Nomura model. The best-fit results of each model are shown in Fig. A.5 (model D1: $1.0 \times 10^{-7}\left(\mathrm{X}_{\text {in }}^{\mathrm{HDO}}\right)$ and $1.0 \times 10^{-11}\left(X_{\mathrm{out}}^{\mathrm{HDO}}\right)$; model D2: $5.0 \times 10^{-8}\left(X_{\text {in }}^{\mathrm{HDO}}\right)$ and $5.0 \times 10^{-12}\left(X_{\mathrm{out}}^{\mathrm{HDO}}\right)$; model D3: $3.0 \times 10^{-8}\left(X_{\text {in }}^{\mathrm{HDO}}\right)$ and $\left.5.0 \times 10^{-14}\left(X_{\text {out }}^{\mathrm{HDO}}\right)\right)$. Comparing all spectra, we find that model D3 produced intensity similar to the Rolffs model in high-excitation lines and less emission in the $893 \mathrm{GHz}$ line. However, the 464 and $893 \mathrm{GHz}$ lines are optically thicker $(\tau=2.9$ at $464 \mathrm{GHz}$ and $\tau=29.6$ at $893 \mathrm{GHz})$ than the lines produced by the Rolffs model. The high opacities result in self-absorption in the 464 and $893 \mathrm{GHz}$ spectra (less emission). Moreover, results (464 and $893 \mathrm{GHz}$ spectra) simulated with models D1 and D2 are very similar to the results produced with the original Rolffs model. Thus, we find that these models 


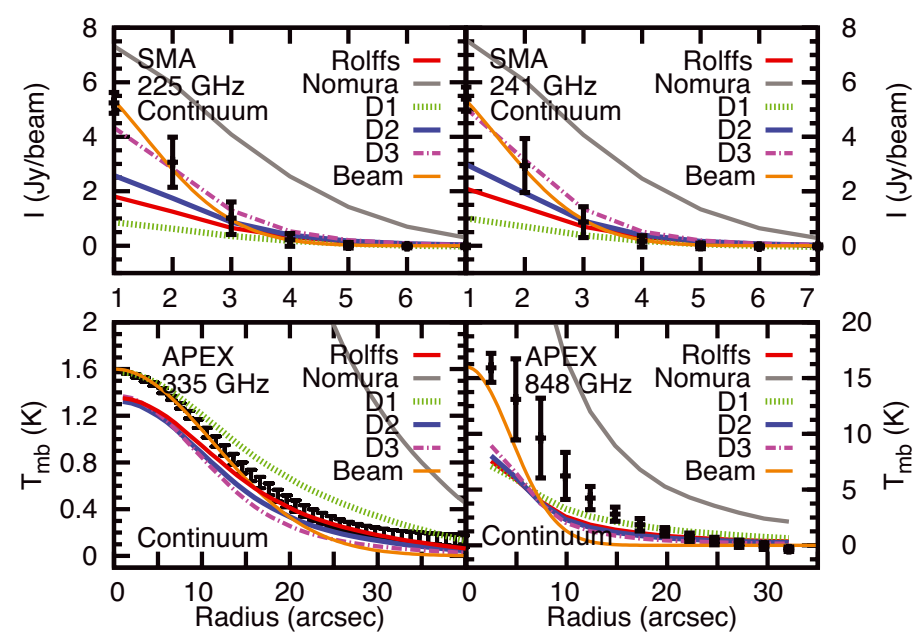

Fig. A.4. Radial profiles of the continuum emission at 225 (SMA), 241 (SMA), 356 (LABOCA, Rolffs et al. 2011), and $848 \mathrm{GHz}$ (SABOCA, Wyrowski et al., in prep.) The modified models are overlaid in different colors (model D1 - green, D2 - dark-blue, and D3 - purple). The red and grey lines are reproduced profiles from the original Rolffs' and Nomura's models. The beam is shown as a orange dashed Gaussian. The errorbars represent only the deviation from a circular shape.
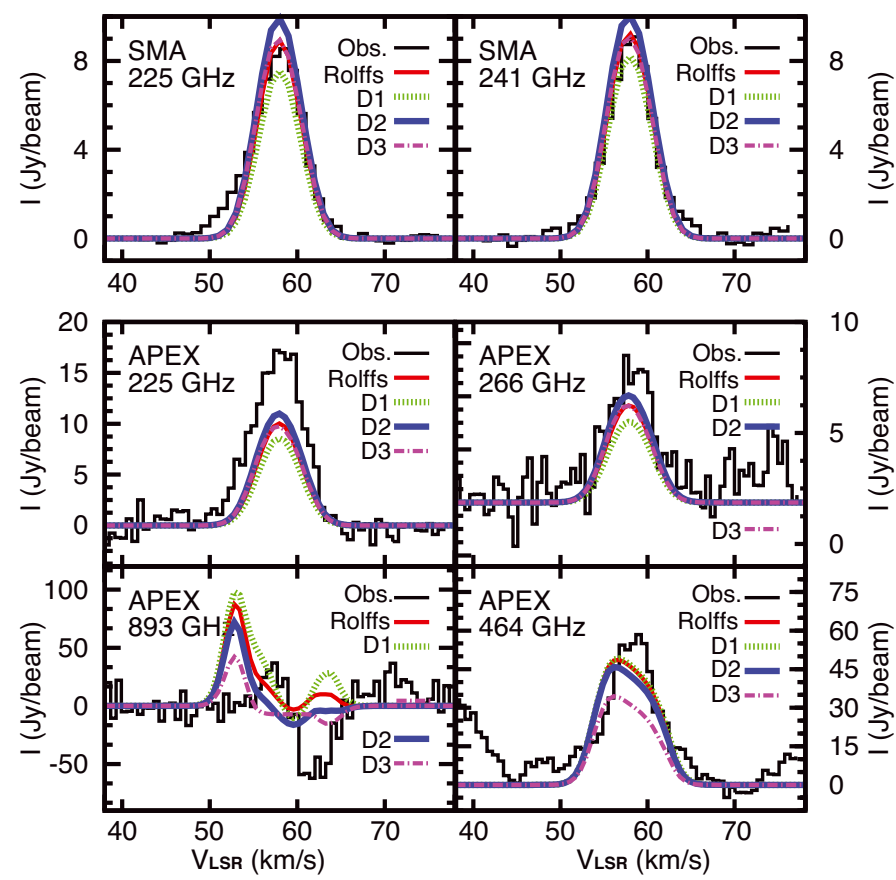

Fig. A.5. Comparison of the observed spectra with the best-fit results of 3 different models with modified density profiles (Fig. A.3). The red lines are the best-fit model of the original Rolffs profile. The observed and simulated spectra at $241 \mathrm{GHz}$ are very similar to those at $225 \mathrm{GHz}$; therefore, only the spectra at $225 \mathrm{GHz}$ are plotted here.

are not significantly better than the original the Rolffs model. In other words, the fits cannot be improved by simply modifying the density profile.

\section{A.3. Modified temperature profile}

To decrease the emission in $893 \mathrm{GHz}$ spectra and maintain the emission in high-excitation lines $(225,241,266 \mathrm{GHz})$, modifying the temperature profile is a possible way. We increased the

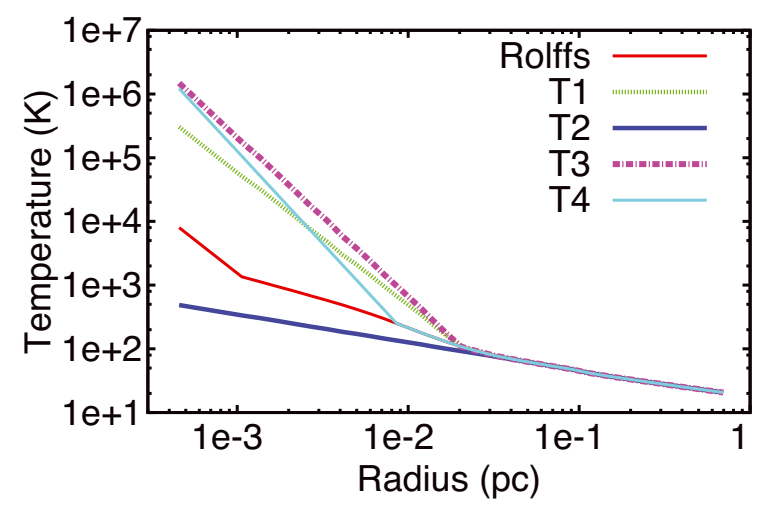

Fig. A.6. Comparison of the temperature profiles of the four different modified models (blue, green, light blue and purple) and the one of the original Rolffs model (red).

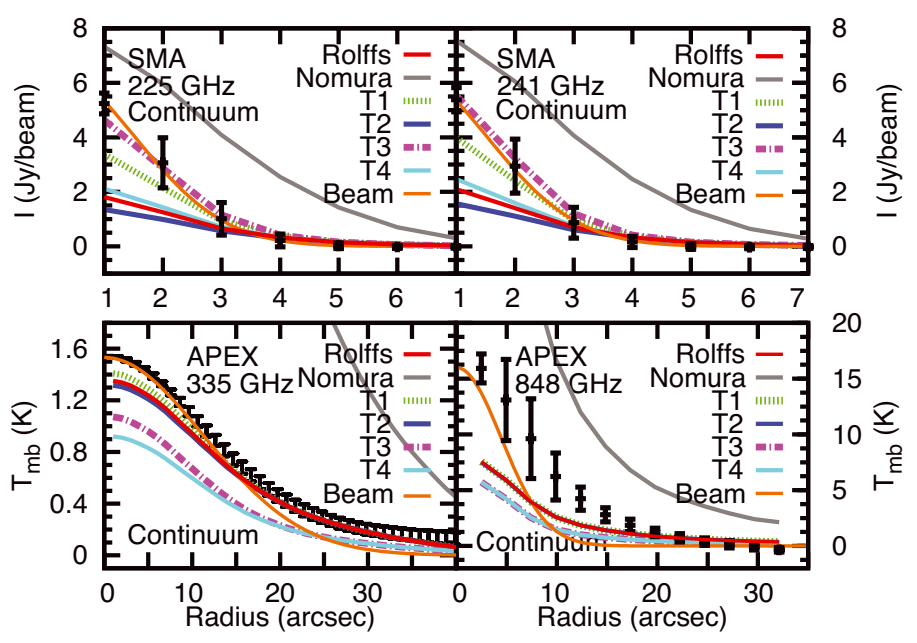

Fig. A.7. Same as Fig. A.4, but for the modified temperature models.

temperature in the very inner region (models T1, T3, and T4) of the core and decreased the fractional abundance in the inner region of the core. The modified and original temperature profiles are shown in Fig. A.6. Model T2 contrasts with other models.

Like for changing the density profile, modifying the temperature profile also influences the continuum emission. Figure A.7 compares the radial profiles of the continuum emission of different models in the four bands. Again, we find that the Rolffs model and all modified models are superior to the Nomura model in all bands. For the 225 and $241 \mathrm{GHz}$ band, model T2 and the Rolffs model fit better, because the dust continuum is less than $25 \%$ of the total continuum flux, implying that increasing the temperature in the central region of the core is a poor solution. In the 335 and $848 \mathrm{GHz}$ bands, T1, T2, and the Rolffs models are superior to others. The indistinguishable continuum at $848 \mathrm{GHz}$ is due to a combination of optical depth and beam dilution effect: the temperature profile is changed only in the very central region of the core where the opacity is high and the beam size is much larger than the scale of the modified region (beam is $\sim 7.4^{\prime \prime}$ at $848 \mathrm{GHz}$ and $2 \times 10^{-2} \mathrm{pc}$ corresponds to $\left.\sim 1.1^{\prime \prime}\right)$. For 241 and $225 \mathrm{GHz}$, the angular resolution is sufficiently high and the opacity sufficiently low to detect the very inner region of the core.

To compare the effect on the spectra, Fig. A.8 shows the fitting results of the different modified models with a fixed HDO fractional abundance in the inner and outer region $\left(\mathrm{X}_{\mathrm{in}}^{\mathrm{HDO}}=6 \times 10^{-8}\right.$ and $\left.\mathrm{X}_{\mathrm{out}}^{\mathrm{HDO}}=5 \times 10^{-12}\right)$. The fitting spectra of 

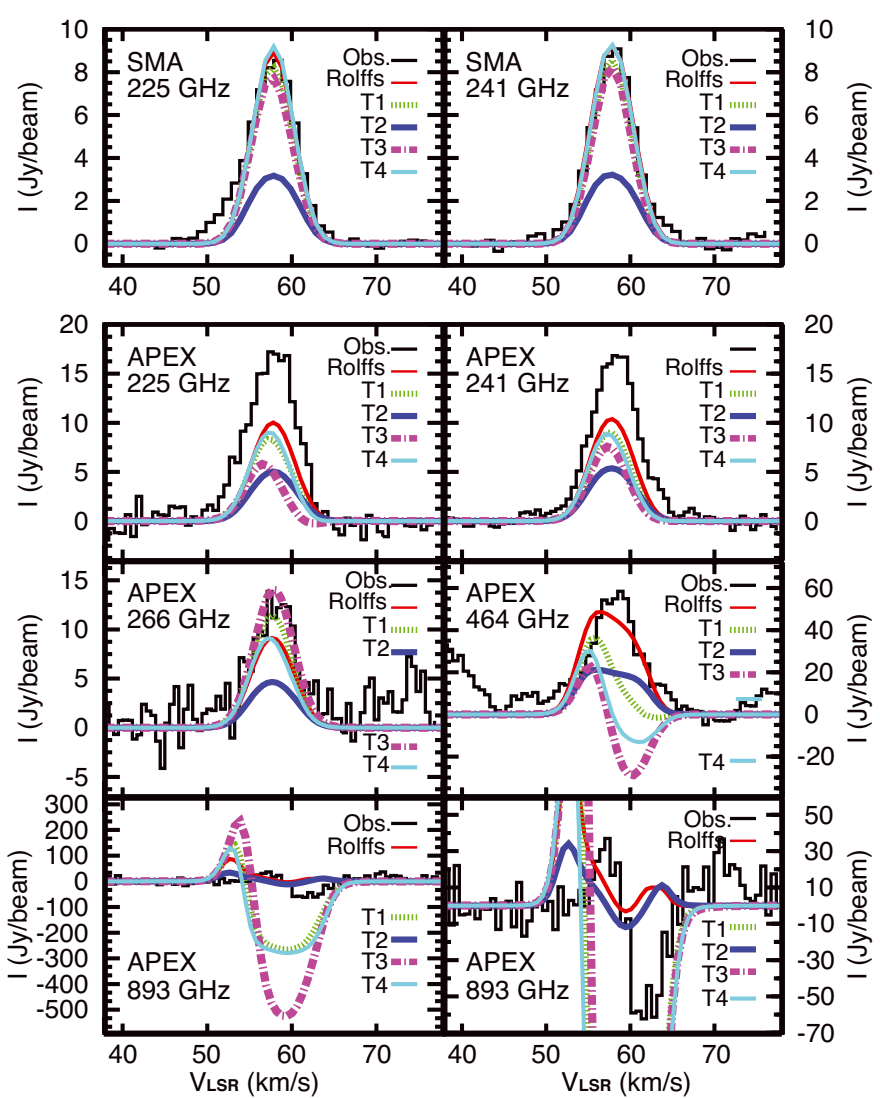

Fig. A.8. Comparison of the observed spectra and those reproduced with the fixed fractional abundances of models T1 to T4. The red lines are the best-fit model with the Rolffs original density profile.

the T1 and T4 models are similar to spectra of the Rolffs model in the high-excitation spectra at 225 and $241 \mathrm{GHz}$, while the T2 model produces less emission in all spectra. However, models $\mathrm{T} 1, \mathrm{~T} 3$, and T4 produce much poorer fits to the two groundtransition spectra, and T2 already becomes optically thick $(\tau=$ 3.4 ) at $464 \mathrm{GHz}$. Hence, simply modifying the temperature profiles does not improve the agreement between the model and the observations. 